\title{
Soziologie der Nachhaltigkeit
}

\section{Herausforderungen und Perspektiven}

Zusammenfassung: Im Diskurs um Nachhaltigkeit fehlt bislang eine spezifisch soziologische Perspektive. Gerade angesichts der Vielfalt des Diskurses kann eine „Soziologie der Nachhaltigkeit“ jedoch unterschiedliche Perspektiven aufeinander beziehen und kritisch auf implizite Prämissen reflektieren. Eine spezifische Herausforderung liegt für die Soziologie darin, sich zu der Problemorientierung sowie zum normativen Charakter der Nachhaltigkeit zu positionieren. Dieser Beitrag macht den Vorschlag, die Perspektivenvielfalt der Soziologie als Stärke zu betrachten, die es angesichts der parallelen Vielfalt der Nachhaltigkeit produktiv zu machen gilt. Als soziologische Zugänge zurNachhaltigkeitsdebattegelten dabei solcheAnsätze, die sich zur Gleichzeitigkeit von Beobachtungs- und Transformationsorientierung verhalten und - ob mit bereichsspezifischem oder gesellschaftstheoretischem Fokus - zu durchaus pluralen Vermittlungsvorschlägen kommen. Explorativ mit Blick auf das Feld einer Soziologie der Nachhaltigkeit werden fünf derartige Vorschläge exemplarisch vorgestellt: doing sustainability, eine feldtheoretische Analyse, ein wissenschaftssoziologischer Beitrag, epistemische Governance und eine gesellschaftstheoretische Reflexion. Wir verstehen dies als Auftakt für eine Diskussion der „Soziologie der Nachhaltigkeit“.
Abstract: This paper proceeds on the assumption that a specifically sociological perspective is lacking in the discourse on sustainability. However, especially in view of the diversity of this discourse, a "sociology of sustainability" may offer new insights by contributing different perspectives and critically reflecting on the implicit premises of the sustainability debate. A specific challenge for sociology is to position itself with regard to the problem-orientation as well as the normative character of sustainability. This contribution proposes to consider the perspective-rich diversity of sociology as a strength, which, as it applies to the parallel diversity of sustainability, can be made very productive. Sociological approaches applicable to the sustainability debate are those that bear upon the simultaneity of observation and orientation of transformation - whether with an area-specific or societal-theoretical focus - and arrive at thoroughly plural mediation proposals. This paper introduces five such proposals as exploratory examples: doing sustainability, a field theoretical analysis, a contribution from the sociology of science, epistemic governance and a societal-theoretical reflection. We understand this as a prelude to a discussion regarding the coming sociology of sustainability. 
Prof. Dr. Anna Henkel ist Professorin für Kultur- und Mediensoziologie an der Leuphana Universität Lüneburg. Forschungsfelder: Sozial- und Gesellschaftstheorie in ihrer Verbindung mit empirischer Forschung, Einbeziehung von Materialität und Dingen in soziologische Untersuchungen sowie empirische Fragestellungen um Nachhaltigkeit, Gesundheit, Verantwortung und Organisation. anna.henkel@leuphana.de

PD Dr. Stefan Böschen ist Senior Research Scientist und Ko-Leiter des Forschungsbereichs Wissensgesellschaft und Wissenspolitik am Institut für Technikfolgenabschätzung und Systemanalyse (ITAS) des KIT. Arbeitsschwerpunkt: Transdisziplinäre Wissenschafts- und Technikforschung. stefan.boeschen@kit.edu

Louisa Firnenburg, B.Sc., ist Studentin des Forschungsmasters Behavioral and Social Sciences an der Rijksuniversiteit Groningen in den Niederlanden. Ihre Forschungsschwerpunkte sind Nachhaltigkeit und Gemeinschaft. l.a.firnenburg@student.rug.nl

Nikolai Drews, M.A. ist seit 2015 wissenschaftlicher Mitarbeiter im Projekt „Reflexive Responsibilisierung. Verantwortung für nachhaltige Entwicklung“ an der Leuphana Universität Lüneburg/Carl von Ossietzky Universität Oldenburg. Arbeitsschwerpunkte: Soziologische Theorie, Systemtheorie, Umweltsoziologie, empirische Sozialforschung. nikolai.drews@leuphana.de

Benjamin Görgen, M.A. ist wissenschaftlicher Mitarbeiter am Institut für Soziologie der WWU Münster. Er arbeitet vor allem zu den Themen Sozialisation, Zivilgesellschaft und Nachhaltigkeit und promoviert zum Thema „Transformationspotentiale gemeinschaftlicher Wohnprojekte im urbanen Raum“. benjamin.goergen@uni-muenster.de

Prof. Dr. Matthias Grundmann lehrt Soziologie am Institut für Soziologie der WWU Münster. Seine Forschungsschwerpunkte sind Sozialisationsforschung, die Soziologie sozialer Beziehungen und Praxis sowie Gemeinschafts- und Nachhaltigkeitsforschung. matthias.grundmann@uni-muenster.de

Dr. Nico Lüdtke ist seit 2015 Postdoc im Projekt „Reflexive Responsibilisierung. Verantwortung für nachhaltige Entwicklung“ an der Leuphana Universität Lüneburg/Carl von Ossietzky Universität Oldenburg. Arbeitsschwerpunkte: Soziologische Theorie (insb. Sozialtheorie), Wissenssoziologie, Wissenschaftssoziologie, qualitative Sozialforschung, historische Soziologie, Wandel der Wissenschaft, Wissenschaftsverantwortung, Transformations- und Nachhaltigkeitsforschung. nico.luedtke@leuphana.de 
Prof. Dr. Simone Rödder ist Juniorprofessorin für Soziologie insbesondere Wissenschaftsforschung am Institut für Soziologie der Fakultät für Wirtschafts- und Sozialwissenschaften an der Universität Hamburg. Arbeitsschwerpunkte: Wissenschaftsforschung, insbesondere Soziologie der Inter- und Transdisziplinarität und Governance von Wissenschaft und Technik im Fachkulturenvergleich, Wissenschaftskommunikation, Öffentlichkeitstheorie, Theorie und Praxis des Wissenschaftsjournalismus. simone.roedder@uni-hamburg.de

Dr. Thomas Pfister ist Leiter der EnergyCultures Nachwuchsgruppe an der Zeppelin Universität in Friedrichshafen.thomas.pfister@zu.de

Björn Wendt, M.A. ist wissenschaftlicher Mitarbeiter am Institut für Soziologie der WWU Münster. Seine Arbeitsschwerpunkte sind Utopieforschung, politische Soziologie und Nachhaltigkeitsforschung. bjoern.wendt@uni-muenster.de

Soziologie und Nachhaltigkeit

Beiträge zur sozial-ökologischen Transformationsforschung

Sonderausgabe I

ISSN 2364-1282

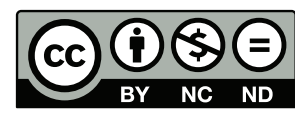

Creative Commons-Lizenz

Herausgeber: Benjamin Görgen, Matthias Grundmann, Dieter Hoffmeister, Björn Wendt

Redaktion: Niklas Haarbusch

Layout/Satz: Frank Osterloh

Anschrift: $\quad$ WWU Münster, Institut für Soziologie Scharnhorststraße 121, 48151 Münster Telefon: (0251) 83-25303

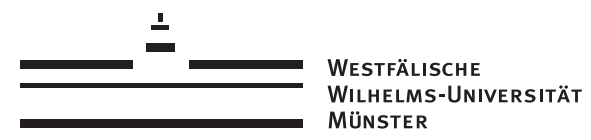

E-Mail: sun.redaktion@wwu.de Website: www.ifs.wwu.de/sun

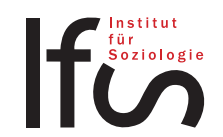




\section{Einleitung:}

Soziologie zwischen Transformation und Reflexion - zur Spezifikation des Verhältnisses der Soziologie zum Nachhaltigkeitsdiskurs ${ }^{1,2}$

Mit zunehmender Dringlichkeit erfolgt eine Diskussion über die langfristige Sicherung menschlicher Existenzgrundlagen. Diese Diskussion wird parallel und mit wechselseitigen Bezügen in vielfältigen gesellschaftlichen Bereichen geführt - im Rahmen der nationalen und internationalen Politik, unter Vertreter*innen von Wirtschaft und Unternehmen, ebenso einer breit verstandenen zivilgesellschaftlichen Öffentlichkeit und nicht zuletzt in der Wissenschaft mit ihren unterschiedlichen disziplinären Foki. Ebenso vielfältig sind die sachthematischen Bezüge. Themen wie anthropogener Klimawandel, Energie, Ernährung, Wohnen und Gesundheit, aber auch Mobilität, Biodiversität und soziale Gerechtigkeit weisen auf die Breite der Diskussion und ihren zugleich fundamentalen und spannungsreichen Charakter hin. Dieses Spannungsfeld wird durch aktuelle Transformationsforderungen verstärkt (WBGU 2011).

Diese Diskussionen zur langfristigen Sicherung menschlicher Existenzgrundlagen erfolgt zum Teil, wenn auch keineswegs ausschließlich, unter dem Stichwort der „Nachhaltigkeit“. Obwohl dieser Begriff nicht einheitlich definiert ist, deutet

1 Dieser Text ist in Fortführung der Vorträge und Diskussionen im Rahmen der Ad-hoc Gruppe "Soziologie der Nachhaltigkeit" auf dem Soziologiekongress 2016 in Bamberg (geleitet von Anna Henkel und Nico Lüdtke) entstanden und sieht sich zugleich als erster Beitrag zu den Diskussionen des gleichnamigen wissenschaftlichen Netzwerks der DFG (Förderzeitraum Januar 2017 bis Dezember 2019) an dem Anna Henkel (Hauptantragstellerin), Thomas Barth, Cristina Besio, Katharina Block, Stefan Böschen, Sascha Dickel, Matthias Groß, David Kaldewey, Gesa Lindemann, Nico Lüdtke, Sighard Neckel, Thomas Pfister, Simone Rödder, Matthias Schloßberger, Björn Wendt sowie als ständiger Gast Jens Jetzkowitz beteiligt sind.

2 Anna Henkel sich in ihm ein zentraler Zug dieser Debatte an, nämlich ihre Normativität sowie Handlungs- und Wertorientierung: Ob Klimawandel, Energie oder Mobilität, stets geht es handlungsorientiert um die Frage, wie hinsichtlich dieses Gegenstands ein Umgang aussehen kann und soll, der mit einer langfristigen Sicherung menschlicher Existenz kompatibel ist. Verschiedene wissenschaftliche Disziplinen tragen in dieser Hinsicht ihre speziellen Sichtweisen in der Debatte bei. So schlagen beispielsweise Ökonom*innen eine Vermarktlichung externer Effekte etwa im Sinne des Emissionshandels vor; Politikwissenschaftler*innen untersuchen spezifische Governance-Mechanismen; Kommunikationswissenschaftler*innen eruieren die Rolle traditioneller und neuer Medien für nachhaltigere individuelle und kollektive Lebensstile, Naturund Ingenieurswissenschaftler*innen entwickeln Innovationen, die konkrete Herausforderungen technisch lösen, etwa im Sinne alternativer Energielieferanten; Erdsystemwissenschaftler*innen schließlich untersuchen im Sinne von Grundlagenforschung globale Umweltveränderungen.

Blickt man auf diese Lage der Dinge, so fällt auf, dass die Perspektive der Soziologie in der Nachhaltigkeitsdebatte spürbar fehlt. Zwar liegt vor allem mit der Partizipations- und Akzeptanzforschung durchaus eine sozialwissenschaftliche Expertise für konkrete Fragestellungen im Nachhaltigkeitsbereich vor. Doch liegt der Schwerpunkt darauf, sozialwissenschaftliche Forschungsmethoden für eine Begleitforschung im Kontext konkreter Nachhaltigkeitsunterfangen anzuwenden - etwa mit Bezug auf konkrete Bürger*innenbeteiligungen (Striegnitz 1995; Renn et al. 1998; Baranek/Günther 2005; Geis 2005; Newig/Fritsch 2009), bei der Beurteilung von Bürger*innenbeteiligung hinsichtlich politischer oder rechtlicher Ziele (Bingham 1986; Coglianese 1997) oder der Untersuchung von Akzeptanz in konkreten Fällen (Würtenberger 1996; Lucke 1997; Hampel 1999; Conrad 2005; Ullrich 2008), 
wobei nur selten auf eine mögliche Instrumentalisierung oder Widersprüchlichkeit reflektiert wird (Newig/Fritsch 2011). Die Soziologie tritt in diesem Forschungsfeld kaum als Perspektive in Erscheinung, die den verhandelten Gegenstand eigenständig rahmt. Obwohl die Partizipationsund Akzeptanzforschung der umfangreichste Bereich sozialwissenschaftlicher Forschung mit Nachhaltigkeitsbezug ist, bietet er daher kaum Ansatzpunkte, um Nachhaltigkeit als soziologischen Gegenstand zu erschließen.

Was die Soziologie bislang nicht sichtbar in die Nachhaltigkeitsdebatte eingebracht hat, ist ihre genuin disziplinspezifische Perspektive: die Reflexion auf den gesellschaftlichen Rahmen von handlungsorientierten Maßnahmen zur langfristigen Sicherung der menschlichen Existenzgrundlagen. Gerade die sozial- und gesellschaftstheoretische Erschließung von Nachhaltigkeit als Gegenstand ist jedoch erforderlich, um die skeptische Haltung weiter Teile der Soziologie im Sinne einer soziologischen Nachhaltigkeitsforschung produktiv zu machen: Aus ihrer reflexiven Position heraus hat die Soziologie das Potential, implizite Paradoxien der Nachhaltigkeitsdebatte jenseits der expliziten Normativität des Konzepts aufzudecken und sowohl Interessenpolitik als auch Interventionshemmnisse aufzuzeigen. Dies gelingt, indem die Perspektivenvielfalt der Soziologie für die Nachhaltigkeitsdebatte eingesetzt wird.

Ausgangspunkt der Diskussion um eine „Soziologie der Nachhaltigkeit“ ist daher die Vermutung, dass die zentrale Kompetenz der Soziologie, nämlich die Verortung sozialen Handelns in einem gesellschaftlichen Kontext, erforderlich ist, um die dezidiert handlungsorientierte Debatte sozial- und gesellschaftstheoretisch zu informieren. Dies erscheint nicht zuletzt deshalb erforderlich, weil trotz umfangreicher Debatten, Maßnahmen und Eingriffen deren Erfolg bislang ausbleibt (Brand 2002; Feindt/Newig 2005; Barry
2012). Die ungebrochene Nicht-Nachhaltigkeit individuellen wie kollektiven Handelns ist ebenso erklärungsbedürftig wie eine nähere Bestimmung des Nachhaltigkeitsbegriffs selbst erforderlich scheint. Indem die Soziologie „Handlung“ hinterfragt, theoretisch unterschiedlich reflektiert und auf Konzepte von Gesellschaft bezieht, ist es möglich, Interaktionen und Folgeprobleme handlungsorientierter Maßnahmen zu beobachten, damit unmittelbar umsetzungsorientierte Konzepte nicht zu kurz greifen.

Die Soziologie bietet für eine solche konzeptionell angeleitete gesellschaftstheoretische Verortung unterschiedliche Möglichkeiten: Neben der Umweltsoziologie haben die gesellschaftstheoretisch orientierte Wissenschaftsforschung, das interdisziplinäre Feld der Science and Technology Studies sowie die Ansätze der Kritischen Theorie, der Systemtheorie, der Praxistheorie und der sozialökologischen Forschung Konzepte und Forschungsperspektiven entwickelt, die sich mit Blick auf eine Soziologie der Nachhaltigkeit aufeinander beziehen und verbinden lassen. Dazu gehören etwa das Verhältnis von Natur und Gesellschaft, das Verhältnis von Wissen und Risiko, das Verhältnis von sozialer Eigendynamik und Steuerungsintentionen sowie Maßstäbe gesellschaftlicher Kritik (Henkel 2016a). Folgende Ansätze zeichnen sich in diesem Sinne ab:

Mit einem Fokus auf Verhältnisse von Natur und Gesellschaft finden sich umweltsoziologische Überlegungen bereits bei den Klassikern der Soziologie (Groß 2001). Zu einer eigenständigen Forschungsrichtung entwickelt sich die Umweltsoziologie in den 1970er Jahren jedoch eher als Folge politischer Debatten und zivilgesellschaftlicher Initiativen, denn als Treiber derselben. In dieser Zeit entstehen klassische Schriften der Umweltsoziologie (Buttel 1976; Catton/Dunlap 1978), deren Schwerpunkt darauf liegt, die bislang in der Soziologie wenig beachtete Kategorie der Natur einzuführen. Im weiteren Verlauf werden 
soziologisch-theoretische Perspektiven für die Frage nach gesellschaftlichen Naturverhältnissen und die Ursachen und Folgen von ökologischen Problemen vielfältig fruchtbar gemacht, was zahlreiche jüngere Überblickswerke reflektieren (Diekmann/Jaeger 1996; Groß 2011; Brand 2014; Lockie et al. 2014). Allerdings bleiben eine systematische Auseinandersetzung mit Nachhaltigkeit und eine Selbstverortung in der Nachhaltigkeitsdebatte weitgehend aus.

Ganz ähnlich behandelt die Wissenschafts- und Technikforschung für die Nachhaltigkeitsdebatte zentrale Aspekte wie Technikgenese (Kowol 1998), Wissensproduktion (Knorr Cetina 1981; Latour/ Woolgar 1986), Nichtwissen (Wehling 2006), Risiko (Beck 1986), Governance (Schuppert/ Voßkuhle 2008) oder das Mitwirken der Technik im Sozialen (Rammert/Schubert 2006; Rammert 2007) sowie mit der modernen Verknüpfung von Innovation und Risiko einhergehende Paradoxien einer Wissensregulierung (Bora et al. 2014). Doch wird auch hier in der Regel darauf verzichtet, die eigene Forschung in der Nachhaltigkeitsdebatte zu situieren.

Schließlich befasst sich die Soziologie auf kulturund wissenssoziologischer Ebene mit Aspekten wie der Kontingenz von Sozialitäts- und Naturkonzeptionen (Lindemann 2009; Descola 2011; Lüdtke 2015), verschiedenen Wissensformen (Pieper 1979; Schützeichel 2007) und ihrer sozialen Einbettung sowie nicht zuletzt gesellschaftlichem Wandel und der gesellschaftlich überhaupt möglichen Resonanz auf ökologische Bedrohungen (Luhmann 1986). Gesellschaftliche Selbstgefährdung auch auf einer sozialen Ebene durch wachsende soziale Ungleichheit, eine (Selbst-)Überforderung der Subjekte (Neckel/ Wagner 2013; Neckel 2015) und ihrer Verantwortungsfähigkeit (Henkel 2013/2014; Henkel/ Åkerstrøm-Andersen 2013/2014) stehen ebenfalls in diesem Kontext. Doch auch hier gilt: All dies wird zunächst nicht aktiv in die Nachhaltigkeitsdebatte eingebracht.

Erst in jüngster Zeit entstehen Ansätze, Nachhaltigkeit trotz, jenseits oder wegen der normativen Konnotation der Debatte aus soziologischer Perspektive als eigenständigen Gegenstand in den Blick zu nehmen und theoretisch einzuordnen. In der Perspektivenvielfalt der Soziologie liegt mit Blick auf die Entwicklung einer Soziologie der Nachhaltigkeit eine Stärke und zugleich eine Herausforderung: Die Stärke ist, dass je nach Perspektive unterschiedliche Aspekte ins Zentrum der Analyse gerückt werden können. Angesichts der Heterogenität der im Kontext von Nachhaltigkeitsfragen zusammenwirkenden Aspekte ist diese Möglichkeit des Perspektivwechsels von Vorteil. Zugleich liegt in dieser Perspektivenvielfalt jedoch die Herausforderung für die Soziologie, als eigenständige Stimme im Nachhaltigkeitsdiskurs sichtbar zu werden.

Das Anliegen einer Diskussion um eine „Soziologie der Nachhaltigkeit“ ist daher, unterschiedliche Zugriffe der Soziologie auf Nachhaltigkeit aufeinander zu beziehen und sowohl Unterschiede als auch (im Sinne einer Synthese) gemeinsame Schwerpunkte zu identifizieren und produktiv zu machen. Zum jetzigen Zeitpunkt bestehen gute Rahmenbedingungen für den Erfolg dieses Unterfangens: In der Nachhaltigkeitsdebatte wird seit Neuestem, etwa seit dem Pariser Klimaschutzabkommen, ein Bedarf an sozialwissenschaftlichen Perspektiven als Desiderat thematisiert; zugleich entstehen seit kurzem - nicht zuletzt auf der Ebene des wissenschaftlichen Nachwuchses und im Kontext von Drittmittelaktivitäten - Initiativen, soziologische Zugangsweisen und Konzepte für konkrete Fragestellungen im Bereich der Nachhaltigkeit fruchtbar zu machen. Die hier aufeinander bezogenen Perspektiven bringen exemplarisch solche aktuellen Initiativen mit ihren unterschiedlichen theoretisch-thematischen Schwerpunkten zusammen und können so einen 
ersten Beitrag zur Integration soziologischer Perspektiven auf Nachhaltigkeit leisten sowie zur Sichtbarkeit der Thematik in der Soziologie und zur Sichtbarkeit der Soziologie in der Nachhaltigkeitsdebatte beitragen.

Die Zusammenstellung und Anordnung der hier exemplarisch aufeinander bezogenen Perspektiven trägt der Vermutung Rechnung, dass „Nachhaltigkeit“ als thematischer Gegenstand systematische und neuartige Spezifika aufweist. Während etwa hinsichtlich sozialer Gegenstände wie Familie, Wirtschaft, selbst Emotionen oder Umwelt theoretische und methodische Ansätze im ersten Schritt in analoger Weise auf solche Gegenstände bezogen werden können und so eine Familiensoziologie oder Umweltsoziologie entsteht, so zeichnet sich „Nachhaltigkeit“ eben durch jenen normativen Bezug und jene Handlungsorientierung aus, auf die oben bereits eingegangen wurde; zudem sind die unter dem Stichwort Nachhaltigkeit verhandelt "grand challenges“ genuin selbst multi-referentielle Probleme, indem sie jeweils zugleich eine Vielfalt gesellschaftlicher Bezüge ansprechen.

Für die Soziologie ergibt sich daraus die für dieses Feld besondere Anforderung der Spezifikation des eigenen Verhältnisses zum Nachhaltigkeitsdiskurs. Dies kann angesichts der disziplinären Binnenpluralität nicht für die Soziologie insgesamt erfolgen. Wohl aber sieht sich, wenn es um Nachhaltigkeit geht, jeder einzelne aus der Soziologie stammende Beitrag mit der Herausforderung konfrontiert, mit der Gleichzeitigkeit von Beobachtungs- und Transformationsorientierung umzugehen und diese mit einem potentiell durchaus unterschiedlichen Grad an reflexiver Distanz zu integrieren. So kann es als zentrales Spezifikum von Nachhaltigkeit gelten, einerseits selbst als soziales Phänomen beobachtbar zu sein und dieser Reflektion auch zu bedürfen; andererseits aber eine Transformationsorientierung zu implizieren im Sinne einer Anwendung so- ziologischer Theorie- und Methodeninstrumente zur Beförderung von „Nachhaltigkeit“ (was dann wiederum näher zu bestimmen ist). Ein Vorteil der Soziologie der Nachhaltigkeit bei Vorschlägen der Integration dieser beiden Dimensionen mag gerade darin liegen, dass sich die Disziplin nicht auf einen einheitlichen Grad der Distanz insgesamt festlegen muss, sondern die Vielfalt ihrer gültigen paradigmatischen Annahmen nutzen kann, um Nachhaltigkeit als Phänomen der Gesellschaft, deren Teil auch die Soziologie ist, aus unterschiedlichen Perspektiven und mit unterschiedlichen Graden der Distanz in den Blick zu nehmen.

Vorschläge aus der Soziologie zur Integration einer Gleichzeitigkeit von Beobachtungs- und Transformationsorientierung unterscheiden sich zudem darin, inwieweit analytisch eher bereichsspezifisch oder gesellschaftstheoretisch fokussiert sind. So lässt sich der Nachhaltigkeitsdiskurs einerseits nahe der Debatte um ökologische Selbstgefährdung, Risikogesellschaft und etwaige neuartige Formen der Wissensproduktion verorten; andererseits geht es zum Teil sehr direkt um die Frage der Wirksamkeit verschiedener Instrumente mit Blick auf nachhaltige Entwicklung, Formen der Governance oder auch Handlungsspielräume bzw. Verantwortung von Akteuren. Wiederum gilt, dass die Soziologie von der Pluralität ihrer Perspektiven profitiert und es auch kaum zielführend wäre, die gesamte Soziologie allein auf eine Perspektive der Wissensforschung oder der Governance festzulegen. Zur Selbstverortung und zur Systematisierung einer emergierenden Soziologie der Nachhaltigkeit ist aber wiederum die Reflexion auf die eigene Position erforderlich. Soziologische Vorschläge zur - unterschiedlich reflexiv distanzierten - überbrückenden Integration von Beobachtungs- und Transformationsorientierung können also einen eher bereichstheorieorientierten Fokus auf Akteure, Institutionen oder Regulierungsinstrumente wählen, oder eine eher gesellschaftstheoretischen Orientierung, die den 
Fokus auf Wissen, Kommunikation und gesellschaftliche Gesamtdynamik legt.

Das emergierende Feld einer Soziologie der Nachhaltigkeit lässt sich vor diesem Hintergrund ordnen, je nachdem wie die reflexive Distanz zwischen beobachtungs- und transformationsorientierter Perspektive auf Nachhaltigkeit integriert wird und welcher spezifische bereichs- oder gesellschaftstheoretische Zugriff dabei gewählt wird. Um das Spektrum dieses Forschungsfeldes aufzuzeigen, seien im Folgenden explorativ fünf Vorschläge auf der „Landkarte der Soziologie der Nachhaltigkeit" näher vorgestellt:

Im Abschnitt „Doing Sustainability - die soziale Konstruktion der Nachhaltigkeit zwischen mikro- und makrostruktureller Praxis“ wird die Integration von Beobachtungs- und Transformationsorientierung mit einem Schwerpunkt auf der Transformationsorientierung und einer bereichsspezifischen Perspektive verbunden, wobei das Phänomen nachhaltigen Handelns im Mittelpunkt steht. Mithilfe des Modells der human- bzw. sozialökologischen Sozialisationsforschung wird untersucht, wie Nachhaltigkeit praktisch gelebt und auf unterschiedlichen Handlungsebenen konkret hergestellt wird - und welche Bedingungen und Hemmnisse je bestehen. Kooperation und wechselseitigen Fürsorge erscheint hier als Grundlage der Nachhaltigkeit (Abschnitt 1: Louisa Firnenburg, Benjamin Görgen, Matthias Grundmann, Björn Wendt). Auch der anschließende Abschnitt zur „Nachhaltigkeitssoziologie als feldtheoretische Analyse problemzentrierter Wissensregime" verbindet Beobachtungs- und Transformationsorientierung mit einem Transformationsschwerpunkt und einer bereichsspezifischen Perspektive, wobei der gewählte Gegenstandsfokus hier der Aushandlungsraum Wissensregime ist. Wissensregime sind verstanden als regulierte soziale Räume zur Artikulation, Definition und Lösung sozio-technischer Probleme, die feldtheoretisch eine Dynamik der Problemfortpflanzung zu beobachten erlaubt (Abschnitt 2: Stefan Böschen). Der folgende Abschnitt „Was kann der wissenschaftssoziologische Beitrag sein zur soziologischen Reflektion von Nachhaltigkeitsdiskursen?" verbindet Beobachtungs- und Transformationsorientierung mit Fokus auf der Beobachtung und wählt mit der Fokussierung auf das Phänomen der Wissenschaft in einer Gesellschaft der Nachhaltigkeit eine stärker gesellschaftstheoretische Selbstverortung. Der Nachhaltigkeitsdiskurs erwarte vom Wissenschaftssystem sowohl problembezogene als auch inter- und transdisziplinäre Forschungsansätze, was spezifische Anforderungen an die wissenschaftliche Produktion von Wissen stelle (Abschnitt 3: Simone Rödder).

Die wissenschaftssoziologische Beobachtungsperspektive auf spezifische Anforderungen von Nachhaltigkeit an die Wissenschaft ist auf einer "Landkarte“ der Soziologie der Nachhaltigkeit gewissermaßen zwischen den beiden ersten Ansätzen verortet, die eine Integration von Beobachtungs- und Transformationsorientierung mit einem Fokus auf der Transformation und einer bereichsspezifischen Perspektive anstreben und den beiden folgenden Ansätzen, die Beobachtungs- und Transformationsorientierung stärker beobachtungsorientiert unternehmen und dabei eine gesellschaftstheoretische Perspektive wählen. Während die Abschnitte zwei und drei deutlich machen, wie soziologische Perspektiven mit Blick auf nachhaltige Entwicklung fruchtbar gemacht werden können, ohne die spezifische soziologische Distanz und Perspektive zu verlieren und der Abschnitt vier mit dem Fokus auf Wissenschaft bereits eine stärkere Beobachtungsorientierung mit bereichsspezifisch-gesellschaftstheoretischem Fokus auf Wissenschaft verbindet, so geht es den beiden abschließenden Abschnitten darum, Beobachtung und Transformation auf starkem Reflexionsniveau $\mathrm{zu}$ integrieren und Nachhaltigkeit als soziales Phänomen gesellschaftlich diskursiv und funktional zu verorten. 
Der Abschnitt „Nachhaltigkeit als Praxis und epistemische Politik“ verbindet Beobachtungsund Transformationsorientierung mit einem Schwerpunkt auf Beobachtung mit einer gesellschaftstheoretischen Perspektive, indem er Nachhaltigkeit als grundlegendes soziales Phänomen untersucht. Im Mittelpunkt steht die Frage, wie die normativen und handlungsleitenden Aspekte des Nachhaltigkeitsdiskurses entstehen und Legitimation erlangen, was als Forschungsperspektive die Untersuchung mittelfristiger Stabilisierungen nahelegt (Abschnitt 4: Thomas Pfister). Auch der letzte Abschnitt „Gesellschaftstheoretische Reflexion der Nachhaltigkeit" wählt bei der Integration von Beobachtungs- und Transformationsorientierung einen Schwerpunkt auf der Beobachtung und verbindet diesen mit einer gesellschaftstheoretischen Perspektive. Die Funktion des Nachhaltigkeitsdiskurses wird analog zur gesellschaftlichen Funktion der Menschenrechte darin gesehen, funktionssystemischen Vereinnahmungstendenzen Grenzen zu setzen. Analog zum Schutz der Person erfolgt hier ein Schutz der Lebensgrundlagen der Person als verkörpertem Menschen gegenüber rein wirtschaftlicher, rein politischer oder rein wissenschaftlicher Vereinnahmung. Gesellschaftstheoretisch stellt sich zudem die Frage einer etwaigen Entdifferenzierung gesellschaftlicher Funktionsbereiche, die für das Wissenschaftssystem hinsichtlich der Verbreitung transformativer und transdisziplinärer Forschung diskutiert wird (Abschnitt 5: Nikolai Drews, Nico Lüdtke). Der Text schließt mit einem Ausblick auf die möglichen Konturen der Debatte um eine Soziologie der Nachhaltigkeit (Abschnitt 6: Anna Henkel).

\section{Doing Sustainability - Die soziale Konstruktion der Nach- haltigkeit zwischen mikro- und makrostruktureller Praxis ${ }^{3,4}$}

Wie Nachhaltigkeit praktisch gelebt und auf unterschiedlichen Handlungsebenen konkret hergestellt wird und werden kann, bleibt in der Nachhaltigkeitsforschung bis heute oftmals unterbelichtet (M. Grundmann 2016). Ausgehend von einer Kritik an der Vernachlässigung der sozialen Dimension von Nachhaltigkeit (Görgen/ Wendt 2015), entwickeln wir den Vorschlag, auf der Grundlage des Modells der human- bzw. sozialökologischen Sozialisationsforschung(Bronfenbrenner 1981; Grundmann/Kunze 2008) die mehrebenenspezifischen - sich gleichwohl wechselseitig bedingenden - Handlungsbezüge von Nachhaltigkeitsakteuren, -diskursen und -strukturen in den Blick zu nehmen. Auf diese Weise erscheint es möglich, zu einem umfassenderen Verständnis der sozialen Konstruktionsprozesse nachhaltiger sozialer Praxis und ihrer Relevanz für gesellschaftliche Transformationsprozesse zu gelangen. Nachhaltigkeit verweist dabei zunächst auf einen zentralen Aspekt menschlichen Handelns, der ganz basal auf dem Erhalt von intra- und intergenerationalen Sozialbeziehungen und damit verbunden auf Fürsorge und gemeinsamer Wohlfahrt basiert. Grundlage dafür ist die Fähigkeit zur Kooperation und zur Regulation sozialer Konflikte (z.B. aufgrund konkurrierender Interessen, um Ressourcennutzung etc.). Diese baut wiederum auf einer Orientierung an allgemeinen Interessen auf, die sich eben nicht auf die Optimierung von Eigennutz alleine, sondern an wechselseitiger Fürsorge und Sicherung von Wohlfahrt ergibt (Honneth 1995; Etzioni 1996; Etzioni 1997; Tomasello 2016).

3 Louisa Firnenburg, Benjamin Görgen, Matthias Grundmann und Björn Wendt.

4 Für eine ausführliche Darstellung der Überlegungen vgl. Firnenburg et al. (2017). 
Nachhaltigkeit bezieht sich somit ganz grundsätzlich auf eine soziale Handlungsorientierung bzw. auf soziale Kontexte, in die das individuelle Handeln eingebunden ist. Daher schlägt sie sich - je nach betrachteter Handlungsebene - in unterschiedlichen Praktiken nachhaltigen Handelns nieder. Für den Nachhaltigkeitsdiskurs (und ebenso für den damit verbundenen Gerechtigkeitsdiskurs) bedeutet das: es geht um ein Aufeinander-Bezogenes- und Füreinander-Sorgendes-Handeln im praktischen mitmenschlichen Vollzug, das stets mit normativen Setzungen verbunden ist. Diese praktisch-normativen Handlungsvollzüge beziehen sich zwar zunächst vor allem auf jene Handlungskontexte, die für den Erhalt des konkreten Miteinanderlebens und Füreinander-Sorgens bedeutsam sind. ${ }^{5}$ Sie werden zugleich aber auch von soziokulturell hervorgebrachten Organisationsformen sowie gesellschaftlichen Strukturen, Diskursen und Weltanschauungen beeinflusst, die den jeweiligen zeithistorisch verankerten und zivilisatorischen Reflexionsmodus auf das menschliche Zusammenleben repräsentieren.

Damit ist ein anderer, soziologisch gesättigter Begriff der Nachhaltigkeit eingeführt, der sich eben nicht nur als makrostrukturelles Regulativ oder als politische Leitidee begründet, sondern seine Fundierung im konkreten mitmenschlichen Sein erhält, das stets in einem Spannungsfeld zu den normativen Leitideen steht. Hier wird dann auch sichtbar: Nachhaltigkeit schließt an eine anthropologisch begründete gattungsspezifische Handlungsorientierung an, die sich - aus handlungs- und gesellschaftstheoretischer Perspektive - zwischen mikro- und makrostrukturellen sozialen Umwelten verorten lässt. Damit verbunden

5 Zu den praxistheoretischen Implikationen dieses Vollzugsmodells siehe Bongaerts (2007). sind aber auch normative Aspekte einer Gesellschaftlichkeit, die eben diese Wohlfahrt sichert. ${ }^{6}$

Der hier entfaltete Vorschlag lautet zusammenfassend, zwischen unterschiedlichen Ebenen der soziologischen Betrachtung von Nachhaltigkeit $\mathrm{zu}$ unterscheiden und diese nicht nur in Bezug auf die Makrostruktur, sondern aus einer mikrosoziologischen Perspektive auszubuchstabieren. Mehr noch: Bereits die normativ-utopischen Leitbilder der Nachhaltigkeit lassen sich nicht nur als Makroutopie einer nachhaltigen Gesellschaft, sondern auch als Meso-, Mikro- und Individualutopien der Nachhaltigkeit beschreiben, indem sozial-ökologische Leitbilder von Organisationen, Gemeinschaften und einzelnen Personen mit in den Blick genommen werden und im Hinblick auf ihre handlungsleitenden Funktionen untersucht werden (Wendt 2017). Indem sich Nachhaltigkeit ferner in und zwischen sozialen und natürlichen Umweltbedingungen mitmenschlichen Handelns etabliert, lassen sich auch die konkreten Praktiken des nachhaltigen Miteinanders und nachhaltiger Lebens- und Haushaltsführung sozialökologisch verorten. Nachhaltige soziale Praxis ist demnach in vielfältige sozial-ökologische Umwelten eingebettet, die im Folgenden mithilfe eines Mehrebenenmodells näher bestimmt werden. Dieses Modell ist sowohl in der Lage, den Dualismus von Natur bzw. der bio-physikalischen Welt und dem Sozialen aufzufangen, als auch die mehrebenenspezifische Einbindung sozialer Praktiken abzubilden.

Den Ausgangspunkt dieser integrativen Denkfigur bildet eine Heuristik, die im Rahmen der sozial-ökologischen Sozialisationsforschung durch Urie Bronfenbrenner entwickelt wurde (Bronfenbrenner 1981). Die sozial-ökologische Sozialisationsforschung untersucht, ausgehend von einer sozial- und entwicklungspsychologischen

6 Siehe dazu die Debatte zwischen Liberalismus und Kommunitarismus, wie sie bei Honneth (1995) dokumentiert ist. 
Modellierung der Humanökologie in Tradition der Chicagoer Schule, die Parallelität von Ontogenese (Persönlichkeitsentwicklung), Soziogenese (Entwicklung sozialer Beziehungen und sozialer Gruppen) und Historiogenese (Gesellschaftsentwicklung), die sowohl für die individuelle und gemeinschaftliche Lebensführung als auch für die gesellschaftliche Entwicklung von zentraler Bedeutung sind (Grundmann 1999; Grundmann/ Kunze 2008; Grundmann 2013). Wenngleich sich die sozial-ökologische Heuristik von Bronfenbrenner ursprünglich auf die persönliche Entwicklung von Individuen in sozialen Umwelten bezog, wurde diese zu einer Darstellung der komplexen gesellschaftlichen Umweltbeziehungen erweitert und ermöglicht eine mehrebenenspezifische Betrachtung der Wechselbeziehungen zwischen individuellen und kollektiven Akteuren und ihren sozial-ökologischen Umwelten. Diese erweiterte Heuristik dient uns als Grundmodell für ein sozial-ökologisches Analysemodell, mit dem wir zum einen zeigen wollen, wie sich nachhaltige Lebensformen und Praktiken im mikrosozialen System, also den konkreten sozialen Beziehungen innerhalb kollektiver Akteure etablieren und zugleich durch meso-, exo- und makrostrukturelle Bedingungen gerahmt sind (M. Grundmann 2016).

Ziel der Modellierung ist die Darstellung der Umwelteinbindung und Umweltgestaltung kollektiver Akteure und ihrer sozialen Praktiken auf vier Ebenen. Auf der Mikroebene steht zunächst das Innenleben eines kollektiven Akteurs, also die sozialen Beziehungen und gelebten Praktiken im Zentrum des Interesses. Die Mesoebene rückt den Blick auf die direkte Umwelt - sozusagen den Sozialen-Nahraum - des Akteurs, den er mit anderen Akteuren teilt, zu denen er in direktem Kontakt steht. Die Exoebene umfasst hingegen Akteure und Organisationen, etwa Unternehmen, Universitäten, Verbände, Behörden oder Gewerkschaften, die für die unmittelbare Praxis des untersuchten Akteurs zwar bedeutsam sind, an deren Hervorbringung er aber nicht selbst beteiligt ist, sodass in diesem Fall von einer nicht geteilten Umwelt gesprochen werden kann. Die Makroebene umfasst schließlich die Einbindung des kollektiven Akteurs in die allgemeine Gesellschaftsstruktur (etwa das wirtschaftliche, politische oder wissenschaftliche System) und die jeweils damit verknüpften Diskurspraktiken.

Wichtig ist unseres Erachtens dabei, diese verschiedenen Ebenen nicht als voneinander unabhängige Strukturebenen zu betrachten, sondern stets als das Ergebnis sozialer Praxis, die jedoch unterschiedliche Grade der Autonomie von einzelnen Akteuren besitzen und ineinander verschachtelt sind (Berger/Luckmann 1980). Bei alldem sind diese sozialen Umwelten wiederum gerahmt von der natürlichen Umwelt als ursprünglicher Bedingung von Gesellschaft. Einerseits verfügen die skizzierten sozialen Umwelten somit stets über eine natürliche bzw. ökologische oder materiell-physische Dimension, andererseits ist dieser Rahmen der natürlichen Umwelt stets bereits eine vergesellschaftete Struktur, so dass zusammengenommen von hybriden, das heißt sozial-ökologischen Phänomenen auszugehen ist, sei es in Bezug auf das Mikrosystem des kollektiven Akteurs, seine meso- und exostrukturelle Umwelt oder das sozial-ökologische Makrosystem.

Dieses allgemeine Modell lässt sich an einem konkreten Beispiel - in diesem Fall an einem alternativen sozial-ökologischen Wohnprojekt im städtischen Raum - wie folgt explizieren (Abbildung 1).

Die Mikroebene umfasst zunächst die Entwicklung des Beziehungsgefüges und der sozialen Praktiken der Bewohner*innen. Auf der Mesoebene des Projektes können nun jene Akteure abgebildet werden, mit denen das Wohnprojekt direkt interagiert (etwa eine Foodsharing-Initiative, ein Umwelthaus, ein Repair-Café oder ein anderes sozial-ökologisches Wohnprojekt). 


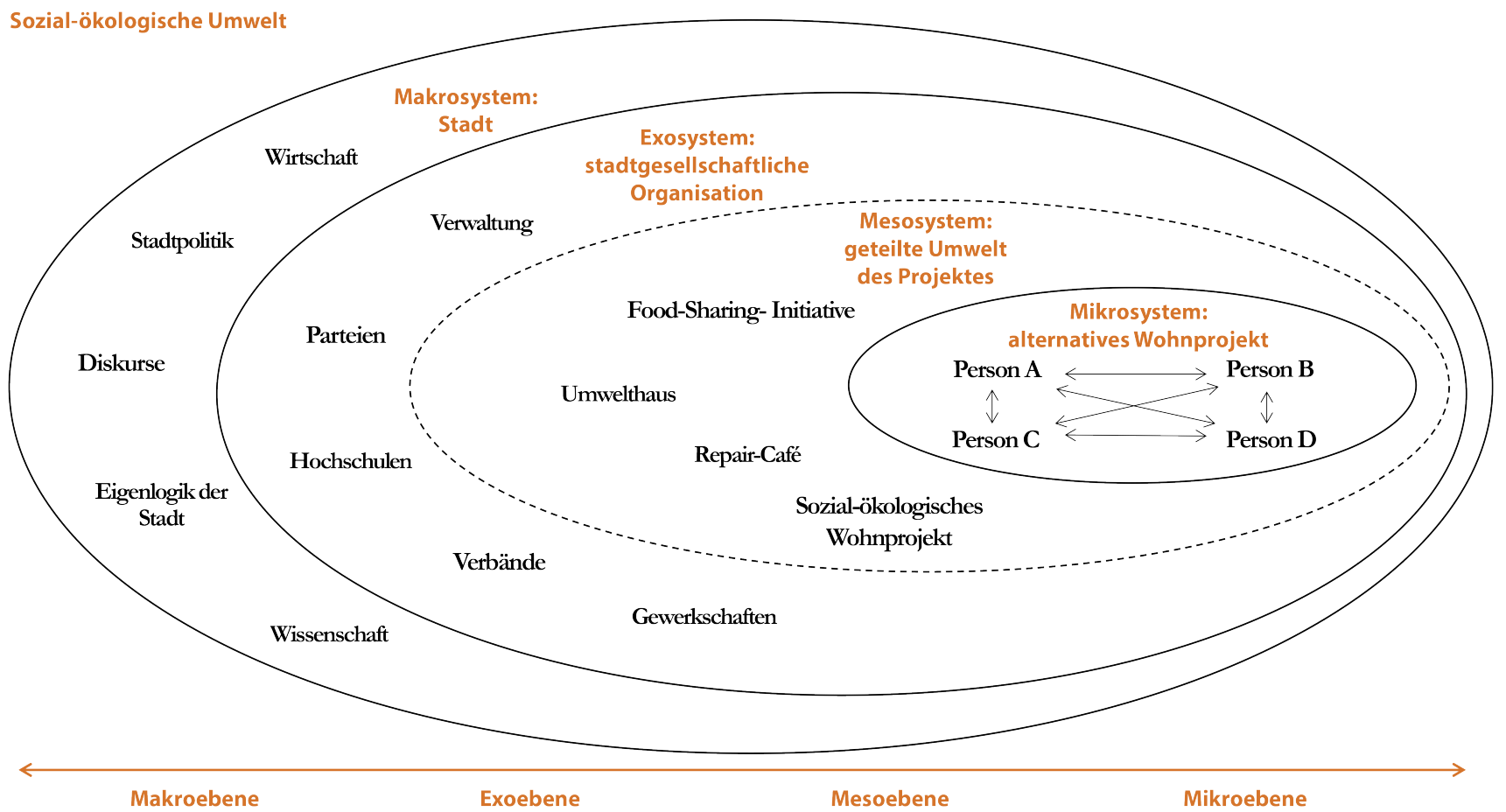

Abbildung 1: Die Sozialökologie eines alternativen Wohnprojektes

Auf der Exoebene können ferner einige stadtgesellschaftliche Organisationen erfasst werden, die zwar einen Einfluss auf die Praxis des Wohnprojektes haben, an denen es selbst aber nicht beteiligt ist, etwa ein Verband, eine Partei, die Universität, die Verwaltung oder eine Gewerkschaft. Wichtig ist dabei zu beachten, dass sich die Verortung einzelner Akteure im Laufe der Zeit zwischen Meso- und Exosystem verändern kann, etwa wenn das Projekt eine Kooperation mit einer städtischen Stelle eingeht, die auf diese Weise Teil das Mesosystem des Projekts wird. Die Makroebene umfasst in diesem Beispiel schließlich darüber hinaus das städtische Makrosystem, also etwa die städtische Wirtschaft, die Stadtpolitik, das städtische Wissenschaftssystem und die damit verbunden Diskurse, die ihrerseits wiederum in größere sozial-ökologische Umwelten eingebettet sind. ${ }^{7}$

7 Die Ebene der Stadt wurde in dem Beispiel als Hauptfokus gewählt, da sie für die Entwicklung des Wohnprojektes zentral ist und einen überschaubare Analyserahmen bietet, um die Wechselwirkungen zwischen den Akteuren und ihrer sozial-ökologischen Umwelt zu untersuchen.
Denkt man nun an die Herausforderung der Erforschung nachhaltiger sozialer Praktiken, hier exemplarisch des sozial-ökologischen Wohnprojekts, so ist es zentral, die Bedingungen und Hemmnisse für die Gestaltung von Nachhaltigkeit auf allen Ebenen und in ihren Wechselverhältnissen $\mathrm{zu}$ analysieren. Darüber hinaus ist von Bedeutung, dass die durch die Umwelteinbindung vermittelten Ressourcen und Restriktionen zwar objektiv erlebt werden, aber zugleich stets sozial konstruiert und somit gestaltet und damit auch gestaltbar sind.

Die Entstehung und Entwicklung nachhaltiger Praktiken solcher Wohnprojekte können in diesem Sinne auf verschiedenen Ebenen und hinsichtlich unterschiedlicher Wirkrichtungen analysiert werden. In Bezug auf die Mikroebene, also das Innenleben des Projekts, kann z.B. untersucht werden, wie sich die gemeinsame soziale Praxis im Projekt unter Berücksichtigung spezifischer situativer Kontexte verändert. Dabei geraten mithilfe des Modells sowohl die Bedeutung der spezifischen Beziehungskonstellation innerhalb des Projektes, als auch die externen Einflüsse, wie 
etwa die Bedeutung städtischer Diskurse und Politiken auf der Makroebene oder spezifischer mit dem Projekt interagierender Akteure - etwa einer Food-Sharing-Initiative - für die Entstehung einer nachhaltigen Ernährungspraxis in den Blick. Auch die Bedeutung materieller und nicht-materieller Aktanten kann mithilfe des Modells aufgeschlüsselt werden. So wäre es möglich zu analysieren, welchen Einfluss die konkrete materielle Ausgestaltung des Projekts auf die soziale Praxis hat, etwa hinsichtlich der Bedeutung gemeinschaftlich geteilter Räumlichkeiten. Auch die Bedeutung verschriftlichter Aktanten auf den verschiedenen Ebenen, etwa das Projektverständnis oder auf der Exoebene ein spezifischer Stadtratsbeschluss kann auf diese Weise analysiert werden. Ebenso ist es möglich, die Effekte der sozialen Praxis des Projekts auf die sozial-ökologische Umwelt zu erfassen. Verändert sich etwa durch die Aktivitäten des Projekts das Quartier, in das es sozialräumlich eingebunden ist? Etwa hinsichtlich einer Umgestaltung bestehender Grünanlagen oder dadurch, dass Räumlichkeiten zur Verfügung gestellt werden, in denen sich die Bewohner*innen des Viertels vernetzten können? Hat dies wiederum Einfluss auf gemeinsame soziale Praktiken? Wirkt das Projekt durch seine Vorbildfunktion und sein Engagement auf die Stadtpolitik ein und bewirkt so Veränderungen lokaler Policies?

All diese Überlegungen sind zunächst erst einmal exemplarisch. Das Modell kann unseres Erachtens jedoch als Heuristik dazu beitragen, die verschiedenen komplexen Wirkzusammenhänge in den Blick zu bekommen, damit sie in der Analyse berücksichtigt werden können. Insbesondere durch vergleichende Untersuchungen - in diesem Fall zu den Praktiken unterschiedlicher sozial-ökologischer Wohnprojekte - ist es möglich, verschiedene Transformationsdynamiken in den Blick zu bekommen; etwa solche eines Projektes, das relativ weitreichende Wirkungen erzielt oder eines Projektes, dessen Transformationsbemühungen scheitern. Schritt für Schritt lassen sich auf diese Weise verschiedene Typen von Umwelteinbettungen und Umweltgestaltungen identifizieren, die Hinweise auf allgemeine Bedingungen und Restriktion bei der Entstehung und Institutionalisierung nachhaltiger sozialer Praktiken liefern können, um diese in eine Theorie der sozial-ökologischen Transformation zu überführen.

\section{Nachhaltigkeitssoziologie als feldtheoretische Analyse pro- blemzentrierter Wissensregime ${ }^{8}$}

Ulrich Beck formulierte auf dem Soziologie-Kongress 2014 in seiner Laudatio auf Zygmunt Bauman, dass es in den meisten soziologischen Theorien zu einem „alternativlosen Fortschreiten und Fortschreiben der Gegenwart" komme, obgleich sich doch die gegenwärtige Welt wieder in eine terra incognita verwandle. Deshalb sei das grundlegende Problem einer „Soziologie der Transformation“ nach Beck das folgende: „Die Theoretisierung von Transformation erfordert eine Transformation der Theorie“ - bzw. genauer des Theorieverständnisses (Beck 2014). Eine Soziologie der Nachhaltigkeit muss demnach eine Theorie von und für Transformationsprozesse sein. Denn Nachhaltigkeit stellt ein umstrittenes Entwicklungsprojekt dar. Zwar gibt es einen breiten Konsens über eine erwartet unsichere $\mathrm{Zu}$ kunftsfähigkeit, die Gefährdung der materiellen Basis oder die Herausforderung globaler Koordination. Dem stehen aber auch ebenso Divergenzen hinsichtlich der Rolle von Bürger*innen/Konsument*innen, der Rolle von Technologie, den Lastenverteilungen oder Transformationsweisen gegenüber. Entsprechend entfacht Nachhaltigkeit nicht Konvergenzen von Deutungen, sondern gerade Dissens und Konflikt (Grunwald 2016).

\footnotetext{
8 Stefan Böschen
} 
In der Debatte um Nachhaltigkeit werden unterschiedliche Formen von Wissen mobilisiert und treffen mitunter konflikthaft aufeinander. Deshalb scheint eine doppelte Perspektive hilfreich, die Wissensprozesse zum einen in den Blick zu nehmen erlaubt, zum anderen aber auch Strukturbildungsprozesse mit erfasst. Dies geschieht hier mit einer spezifischen Lesart des Konzepts Wissensregime (zum Überblick: Wehling 2007), das feldtheoretisch ausgedeutet wird (zum Überblick: Böschen 2016). Danach stellen „Wissensregime (...) regulierte soziale Räume zur Artikulation, Definition und Lösung sozio-technischer Probleme und damit der problemzentrierten Wissensproduktion dar." (Böschen 2016: 43) Nun kann der Begriff sozio-technischer Probleme unterschiedlich gedeutet werden (Büscher/Sumpf 2015). Er verweist in jedem Fall auf den Umstand, dass Problemlösungen auf vorherigen Problemlösungen aufruhen und dass dabei das Ineinanderspielen sozialer und technischer Praktiken entscheidend ist (Edwards 2004). Konzeptionell spezifisch bei dieser Deutung von Wissensregimen ist der Umstand, auf die Artikulation und Lösung von Problemen zu fokussieren und dann eine Dynamik der „Problemfortpflanzung“ zu beobachten. Die Ausdeutung solcher Dynamiken als Wissensregime nimmt den Umstand ernst, dass bei solchen Problemfortpflanzungen ganz unterschiedliche Formen von Wissen und Nichtwissen mobilisiert werden und sie deshalb in ihrer Struktur und Dynamik nur verstanden werden können, wenn man zugleich die Regulierung von Wissen sowie die Herstellung der dabei wirksamen Voraussetzungen in den Blick nimmt.

Um dies zu illustrieren, ist ein Blick auf die Agnotology-Forschung instruktiv. Mittels Problemartikulationen kann gezielt Agenda Setting betrieben werden. (Nicht-)Wissen wird risikopolitisch dazu genutzt, mögliche regulative Eingriffe zu verhindern oder größtmöglich abzuschwächen (Proctor/Schiebinger 2008). Die US-Tabakindustrie verstand es dieser Diagnose nach virtuos, sich
Regulationsansprüchen dadurch $\mathrm{zu}$ entziehen, dass sie die grundsätzliche Bedeutung von Risikohinweisen betonte, zugleich aber eindeutige Evidenz forderte, um Verbote $\mathrm{zu}$ rechtfertigen, denen sie sich dann zu beugen versprach. Jedoch muss eine solche Evidenz im Hinblick auf komplexe Probleme als mehr oder weniger unerreichbar angesehen werden. Die Problemartikulation wirkt zugleich als Perspektivenvorgabe, die den Kampf um Problemartikulation sowie die Problemfestlegung und -lösung zu rahmen versucht. Die gezielte Platzierung von Problemen und damit auch von als angemessen wahrgenommenen Evidenzschwellen stellt nicht nur eine Intervention in die Problembearbeitungsaktivitäten in Wissensregimen hinsichtlich ihrer inhaltlichen Gegenstände dar, sondern prägt ebenso ihre prozessual-methodische Form mit. Im Rahmen von meist vielgestaltigen Problemlösungsgeschichten entwickeln sich schließlich Pfade der Problemlösung in Wissensregimen und strukturieren diese. Stabilisiert werden Wissensregime aufgrund etablierter Denk- und Handlungsmuster sowie institutioneller Regeln und stabiler Akteurskonstellationen. Diese Stabilität ist oft so hoch, dass sie ggf. nur durch Ereignisse aufgebrochen werden, die als Skandal dramatischer Nicht-Einlösung von Problemlösungsversprechen symbolisiert werden können.

Hier kommt die feldtheoretische Deutung von Wissensregimen ins Spiel. Denn die Stabilität von Wissensregimen erscheint als dynamische Stabilität, bei der gleichermaßen Mikro- und Makro-Phänomene eine Rolle spielen und deshalb in den Blick genommen werden müssen. In der gegenwärtigen Debatte sind insbesondere die Feldtheorie von Bourdieu (Bourdieu 1998) sowie die von Fligstein und MacAdam (Fligstein/ McAdam 2012) exponiert. Diese erlauben es, die Relation zwischen Strukturbildung und Akteuren als Prozess auszuleuchten. Jedoch bedarf es spezifischer Verknüpfungen in einem integralen Ansatz, weil Bourdieus Feldtheorie Strukturen 
betont und Akteure unterbelichtet, wohingegen Fligstein/McAdam zwar die Relationen zwischen Akteuren als strukturbildend hervorheben, zugleich die strukturierte Struktur vernachlässigen (Böschen 2016). Ein solcher „integraler“ Ansatz einer Feldtheorie betont den Eigensinn von Akteuren sowie den „Eigensinn“ von Dingen, lotet aber zugleich die Interferenz-Bereiche zwischen autonomen wie heteronomen Polen in Feldern aus. In einer konkreten Situation sind demnach die vielfältig vorhandenen Aufforderungsmomente - in einem erweiterten Sinne von Lewins Objekten mit Aufforderungscharakter (Lewin 1963) - zu erfassen und als Ensemble wirksamer Feldkräfte zu rekonstruieren.

Eine besondere Bedeutung kommt dabei spezifischen Konfigurationen von Akteuren zu, welche wesentlich zur Stabilität von Wissensregime beitragen und damit einerseits die Bearbeitung von Problemen sicherstellen, zugleich aber deren Bearbeitung auf bestimmte Pfade begrenzen. Um die Wirksamkeit solcher Konstellationen von Akteuren in Feldern zu beschreiben, bietet es sich an, Symmetrieachsen in Feldern zu identifizieren. Diese lassen sich als „strukturierte Ensemble von (kollektiven) Akteuren und Dingen [verstehen], welche für die anderen Akteure relevante Aufforderungsmomente im Feld platzieren und darin nicht ignoriert werden können." (Böschen 2016: 152). Solche Symmetrieachsen weisen eine Reihe von Besonderheiten auf. Zunächst einmal lassen sich ganz im Sinne von Figstein/McAdam (2012) darunter die Netzwerke der Etablierten verstehen. Jedoch wirken Symmetrieachsen nicht allein aufgrund der Koalitionsbildung von (kollektiven) Akteuren, sondern diese werden vielmehr durch Dinge stabilisiert, z.B. dann, wenn ausgesuchte (kollektive) Akteure über Apparaturen zur Problemmessung bzw. Problembearbeitung verfügen. In Symmetrieachsen versammeln sich die Etablierten arbeitsteilig. Dass solche Symmetrieachsen oftmals erstaunlich stabil sind, das verdankt sich insbesondere zwei Effekten. Zum einen regeln sie die Aufmerksamkeitsökonomie in Wissensregimen, indem sie nur ausgesuchte Aufforderungsmomente als relevante Aufforderungen markieren und zugleich als bearbeitungsfähig symbolisieren. Zum anderen ermöglichen sie für die meisten Akteure ein Aktivitätsminimum, indem sie als vertrauenswürdige Problemmakler erscheinen. In diesem Sinne lässt sich etwa das „iron triangle“ (Bosso 1987) von Landwirtschaft, Ministerium und Industrie verstehen, das in den USA (aber nicht nur dort) die Pestizideinführung regulierte.

Wie lassen sich diese Einsichten wiederum auf die Theoretisierung von Transformationen beziehen, die als Prozesse Nachhaltiger Entwicklung ausgedeutet werden sollen? Unter dem Blickwinkel einer feldtheoretischen Deutung problemzentrierter Wissensregime werden ganz generell Strukturierungsprozesse im Wechselspiel unterschiedlich strukturierter Feldkräfte aufgeschlüsselt. Dies ist mit Blick auf Nachhaltigkeit von besonderem Belang. Denn Nachhaltigkeit wirkt ja als Generalformel zur Bewertung von Transformationen. Wie kann sie aber in unterschiedlichen Wissensregimen Wirksamkeit entfalten? Welche fördernde oder begrenzende Wirkung nehmen dabei etablierte Symmetrieachsen ein - und durch welche Mechanismen werden diese aufgebrochen, um einer Nachhaltigen Entwicklung eine andere oder deutlichere Kontur zu geben? Die Spezifik der Ausdeutung Nachhaltiger Entwicklung wie deren problemzentrierte (Nicht-)Wirksamkeit kann somit erklärt werden. 


\section{Erwartungen an eine nach- haltige Wissenschaft - das Beispiel Klimaforschung ${ }^{9}$}

Der folgende Abschnitt nähert sich der Frage nach einem Beitrag der Wissenschaftsforschung zu einer genuin soziologischen Perspektive auf Nachhaltigkeit, indem er am Fall der Klimaforschung exemplarisch diskutiert, welche Erwartungen an die Wissenschaft mit dem Nachhaltigkeitskonzept einhergehen. Diese Erwartungen betreffen, so die Ausgangsdiagnose, sowohl die innere Ordnung der Wissenschaft als auch ihr Verhältnis zur Gesellschaft, mithin klassische Themen der Wissenschaftssoziologie. Der Beitrag geht von der Annahme aus, dass Nachhaltigkeitsdiskurse vom Wissenschaftssystem einen sowohl problembezogenen als auch inter- und transdisziplinären Forschungsansatz fordern, dessen Ausgestaltung und Praxis wissenschaftssoziologisch beobachtet und interpretiert werden kann. Ziel dieser Beobachtung ist, die reflexive Dimension von Nachhaltigkeitsdiskursen zu stärken und zwar in Bezug auf die Wissenschaft als Produzent einer Ressource Wissen, von der hier wie sonst in der Gesellschaft die Lösung sozialer-ökologischer Problemlagen erwartet wird.

Zwar werden wissenschaftlich-technische Innovationen in diesen Debatten seit langem als Teil des Problems ausgemacht (Beck 1986), gleichwohl erhoffen sich auch Nachhaltigkeitsdiskurse Lösungen primär von (naturwissenschaftlichem) Wissen (Henkel 2016a). In politischen Bemühungen um das Bewältigen "großer gesellschaftlicher Herausforderungen“ (Wissenschaftsrat 2015) oder gar um eine "große Transformation“, in der die Normativität ebenso wie die Handlungsorientierung und der Steuerungsoptimismus des Nachhaltigkeitskonzepts

9 Simone Rödder. Die Forschung der Autorin wird gefördert durch das Bundesministerium für Bildung und Forschung (DFG EXC 177 CliSAP). zum Ausdruck kommen (WGBU 2011), lässt sich sogar typischerweise ein „evidence-first“-Credo feststellen, also eine Überbetonung und damit auch Überforderung mindestens der (Natur-) Wissenschaft, was ihre Kompetenzen und Zuständigkeitsbereiche betrifft.

Für den Fall der Klimaforschung wird das seit längerem und zunehmend kritisiert, indem der Klimawandel als soziales Problem rekonstruiert und entsprechend sozialwissenschaftliche Expertise zu seiner Lösung in Anschlag gebracht wird, mit angesichts der „Wicked-Problem-Natur“ des Klimafalls zum Teil überraschender Zuversicht in deren Problemlösungskapazität (Stehr/von Storch 1995; Agrawal et al. 2012; Ribot 2014; R. Grundmann 2016).

Klimaforschung kann als prototypisch angesehen werden für ein Forschungsfeld, von dem man sich gesellschaftliche Relevanz verspricht im Sinne von entscheidenden Beiträgen zur Lösung eines realweltlichen Problems (vgl. Abschnitt 3). Entsprechend wird sie förderpolitisch unterstützt: Drei der 43 bislang in der Exzellenzinitiative von Bund und Ländern (DFG 2013) geförderten Exzellenzcluster bearbeiten schwerpunktmäßig Klimathemen. Die Forschungsverbünde wählen dazu programmatisch fachübergreifende Ansätze mit einem Schwerpunkt in den Naturwissenschaften, aber bis hin zur Zusammenarbeit und Integration von Sozial- und Naturwissenschaften.

Der Fall Klimaforschung zeigt, dass sich mit Nachhaltigkeit verbundene gesellschaftliche Erwartungen, die für die Wissenschaft als förderpolitische Anforderungen und Anreizstrukturen wirkmächtig werden, in zweierlei Hinsicht konkretisieren lassen: Erstens, in sachlicher Hinsicht, erwartet man von dieser Forschung eine Lösung für die Klimaproblematik und zweitens, in sozialer Hinsicht, wird ein bestimmter Modus erwartet, dieses Klimawissen herzustellen, nämlich durch Inklusion verschieden spezialisierter Wissenschaftler*innen (Interdisziplinarität) sowie durch 
die Inklusion von Nicht-Wissenschaftler*innen als Praxispartner*innen (Transdisziplinarität). Beide Erwartungen werden im Folgenden kurz erläutert.

$\mathrm{Zu}$ 1. In sachlicher Hinsicht soll die Wissenschaft einer nachhaltigen Gesellschaft eine problembezogene oder themenbezogene Forschung sein, die zur Lösung „großer gesellschaftlicher Herausforderungen“ beiträgt, eine Semantik, die David Kaldewey jüngst treffend als einen „neuen Modus der Konstruktion von Objektivität zwischen Wissenschaft und Politik" charakterisiert hat (Kaldewey 2017; s. auch Kaldewey 2013). Von dieser Forschung erhofft man sich ein bedarfsgerechtes Wissen und gemeint ist damit in sachthematischer Hinsicht ein Wissen, das eine Integration im Sinne einer kognitiven Synthese hinter sich hat. Dieses Wissen gilt im Diskurs als traditionell-disziplinärem Wissen überlegen in vielerlei Hinsicht, namentlich was seine Lösungskapazität für gesellschaftliche Problemlagen und seine „soziale Robustheit“ angeht, also etwa die Akzeptanz mit der dieses Wissen in der Öffentlichkeit rechnen kann (Nowotny, Scott et al. 2001; Nowotny 2005).

$\mathrm{Zu}$ 2. In sozialer Hinsicht folgt aus der Orientierung der Wissenschaft auf gesellschaftliche Themen und Probleme hin die Forderung nach einer stärker inklusiven Forschung. Diese Erwartung richtet sich sowohl darauf, Grenzen innerhalb des Wissenschaftssystems zu überwinden (Interdisziplinarität), als auch darauf, Nicht-Wissenschaftler*innen in Forschungszusammenhänge einzubeziehen (Transdisziplinarität). Deshalb wird der Klimaforschung nicht nur die Zusammenarbeit über Fächergrenzen innerhalb der Wissenschafthinweg nahegelegt, sondern auch Kooperationen mit Pra- xispartner*innen in Politik und Verwaltung, in der Wirtschaft und in der Zivilgesellschaft. ${ }^{10}$

Nachgerade die transdisziplinäre Forschung gilt in Nachhaltigkeitsdiskursen als der Forschungsmodus der Nachhaltigkeit und wird aus Sicht der Wissenschaft häufig als Entdifferenzierung interpretiert(vgl.Abschnitt5). Beispielhaft beschreiben Häberli und Kollegen in der Zürich-20oo-Definition Transdisziplinarität als eine neue Form des Lernens und des Problemlösens angesichts komplexer gesellschaftlicher Herausforderungen, die eine Kooperation zwischen Gesellschaft und Wissenschaft notwendig machen (Häberli et al. 2001, siehe auch Grunwald 2016; Jahn et al. 2012). Transdisziplinäre Forschung im Sinne von Problemlösung und Inklusion implementiert das wissenschaftspolitische Leitbild des Nachhaltigkeitsdiskurses in beiden oben genannten Hinsichten: Sachthematisch wird die Integration von Wissensbeständen angestrebt, um Wissen zu generieren, dessen Legitimation sich aus seiner gesellschaftlichen Relevanz und Problemlösungskapazität ergibt. In der Sozialdimension geht es um die Inklusion von Nicht-Wissenschaftler*innen als Praxispartner*innen. Damit wird Partizipation $\mathrm{zu}$ einer Legitimationsressource (Maasen et al. 2006).

Wie sich Wissenschaft im Modus der Transdisziplinarität und Problembezogenheit ausgestaltet und inwiefern ihre Funktions- bzw. Leistungsbeziehungen zur Gesellschaft bzw. zu anderen Funktionssystemen dadurch verändert werden, sollte Gegenstand detaillierter ethnografischer und vergleichender Studien sein. In einem

10 Um die Projektpartner*innen aus der Praxis zusammenzufassen, wird in der Literatur fachübergreifend vorrangig der Stakeholder-Begriff (deutsch: Anspruchs- bzw. Interessenträger oder -gruppen) genutzt, der ursprünglich aus der Ökonomie stammt. Da dieser Begriff jedoch auch den*die Wissenschaftler*in als Akteur mit bestimmten Interessen einschließt, haben wir den Begriff des „Praxispartners" vorgeschlagen, um außerwissenschaftliche Akteure zu bezeichnen (Brinkmann et al. 2015, Kapitel 3 und Schlussfolgerungen). 
Literaturüberblick $\mathrm{zu}$ transdisziplinären Forschungsansätzen konnte gezeigt werden, dass der Forschungsmodus der Transdisziplinarität mit zwei grundsätzlichen Spannungsfeldern konfrontiert ist, bezüglich derer Wissenschaft und Praxis Gegenpole darstellen, d.h. dass ein Wert nicht ohne Verlust des anderen zu maximieren ist (Brinkmann et al. 2015). Ein erstes Spannungsfeld ergibt sich zwischen der gewünschten Problembezogenheit und Handlungsorientierung einerseits und der Anschlussfähigkeit des Wissens innerhalb der Wissenschaft andererseits. Zweitens zeigt sich eine Spannung zwischen der Inklusion von Praxispartner*innen und der möglichen Komplexität der Forschungsmethoden. Hier gilt, dass höhere Inklusivität mit weniger Komplexität einhergeht und umgekehrt. Ein weiterer forschungswürdiger Aspekt ist, dass transdisziplinäre Forschung, Reallabore und Realexperimente als Semantiken Anleihen machen bei der kognitiven Autorität der Wissenschaft, obwohl es gleichzeitig gerade darum geht, die Selbstbezüglichkeit traditioneller Wissensproduktion (des Modus I, Gibbons et al. 1994) zu überkommen, indem die sachliche und soziale Schließung des Wissenschaftssystems - die dominierende Tendenz seit Beginn der disziplinären Differenzierung - in Frage gestellt wird. Es darf deshalb als eine offene Frage gelten, wie sich transdisziplinäre Forschung in gesellschaftliche Problemlagen einerseits und wissenschaftliche Kommunikationszusammenhänge andererseits einbringt und es braucht dazu, auch und gerade seitens der Soziologie, theoriegeleitete empirische Forschung.

\section{Nachhaltigkeit als Praxis und epistemische Politik ${ }^{11}$}

Die im Folgenden entwickelte Perspektive untersucht Nachhaltigkeit als grundlegend soziales Phänomen. Das besondere Interesse dieses Ansatzes gilt den Charakteristiken, historischen und politischen Prozessen hinter der Entstehung zweier eingangs erwähnter zentraler Elemente dieses Begriffs: seiner Normativität und Handlungsleitung. Die Bedeutung des Nachhaltigkeitskonzeptes ergibt sich ja weder aus dem Begriff selbst noch aus den vielfältigen geologischen, physikalischen, chemischen, biologischen, politischen oder ökonomischen Problemlagen, die in diesem Kontext zur Verhandlung kommen können. Insofern stellt sich die Frage, wie seine normativen und handlungsleitenden Aspekte in die Welt kommen und als wahr, relevant und legitim anerkannt werden.

$\mathrm{Zu}$ diesem Zweck unternimmt die Diskussion eine tour de force durch die Wissensgeschichte des Nachhaltigkeitsbegriffs in drei Schritten. Darauf aufbauend folgt eine konzeptionelle Scharfstellung der sozialwissenschaftlichen Nachhaltigkeitsforschung auf die Untersuchung konkreter Problemanalysen, Lösungsansätze, Technologien, Methoden und Materialitäten im Nachhaltigkeitskontext, in deren Rahmen Wissen und Ordnung koproduziert werden. Insbesondere plädiert der Beitrag für die Untersuchung mittelfristiger Stabilisierungen aus letztgenannten Elementen, deren Entstehungsmechanismen und Öffnungsmöglichkeiten.

\section{Drei Phasen der Nachhaltigkeit}

Bedenkt man, in welch kurzer Zeit sich der Begriff der Nachhaltigkeit in den globalen wissenschaftlichen, politischen und wirtschaftlichen

11 Thomas Pfister. Die Forschung des Autors wird gefördert durch das Bundesministerium für Bildung und Forschung (FKZ: 01LN1312A). 
Diskursen und Institutionen etabliert hat, muss dies auf jeden Fall als eine enorme Erfolgsgeschichte gesehen werden - zumal es sich bei der Nachhaltigkeit um eine plurale und vieldimensionale Konzeption handelt, die sich bislang allen Definitionsversuchen immer wieder entzogen hat. Gleichwohl lassen sich in diesem so fluiden wie hybriden Kontext immer wieder Elemente und Phasen größerer Stabilität feststellen, die im Folgenden anhand weniger symptomatischer Ereignisse kurz umrissen werden sollen. ${ }^{12}$

1. Die Entdeckung der Ökologie: Ein wichtiger Grundstein für die Entwicklung des konzeptionellen Rahmens der Nachhaltigkeit wurde schon gelegt bevor der Begriff selbst explizit verwendet wurde: dass es sich bei der Erde um ein so eng zusammenhängendes wie fragiles System handelt auf das die Menschheit massiven (negativen) Einfluss ausübt. Besonders symptomatisch für diese Vorstellung ist der Bericht „Limits to Growth“ (Meadows et al. 1972) an den Club of Rome, der dieses Bild mit Hilfe von, für damalige Zeit bahnbrechenden, Methoden der Computersimulation produzierte und in vielen Dimensionen der Nachhaltigkeitsdebatte (z.B. der Bedeutung von Modellen und Szenarien) bis heute nachwirkt. Diese ökologische Perspektive wird zum einen dadurch charakterisiert, dass sie stets auf große Datenmengen, komplizierte analytische Methoden und die Vermittlung wissenschaftlicher Expert*innen angewiesen ist. Zum anderen stellt sich die menschliche Existenz aus diesem Blickwinkel zunehmend als Bedrohung für die globalen Ökosysteme dar, was einen umgehenden und radikalen Wandel sozialer Praktiken, v.a. im Zusammenhang mit Ressourcenverbrauch und Schadstoffausstoß notwendig macht. Kontroversen sind wissenschaftliche Auseinandersetzungen, oft um Methoden; der Blick auf die Diversität sowie die Ungleichheiten menschlicher

12 Im Folgenden bezieht sich die Darstellung auf die detailliertere Studie von Pfister, Schweighofer und Reichel 2016.
Lebenssituationen ist dagegen verstellt. U.a. mit der UN Umweltkonferenz in Stockholm zieht die ökologische Perspektive auch in die globale Politik ein, mit den Regierungswechseln in den USA und dem Vereinigten Königreich kommt dies aber auch zu einem jähen Ende.

2. Nachhaltige Entwicklung: Zu Beginn dieser Phase des langsamen Übergangs steht keine wissenschaftliche Untersuchung, sondern ein politischer Kompromiss, der durch den sogenannten Brundtland-Bericht (WCED 1987) und den Earth Summit 1992 in Rio de Janeiro in die globalen politischen Institutionen eingeschrieben wird. Schutz der Umwelt, wirtschaftliche Entwicklung und menschliches Wohlbefinden stehen nicht mehr im Widerspruch, sondern werden unter dem neuen Leitbegriff der nachhaltigen Entwicklung als grundsätzlich vereinbar konzipiert. Der Diskurs ist von der wissenschaftlichen Sphäre in die politische gewechselt, das oberste Ziel ist nun globale Gerechtigkeit, insbesondere für die Ärmsten der Welt. Im Verlauf der nächsten 15 Jahre breitet sich der Begriff der nachhaltigen Entwicklung rasant auf alle politischen Ebenen sowie in den Welten der Zivilgesellschaft, der Wissenschaft und der Unternehmen aus. Allerdings gerät der ursprünglich normativ-politische Fokus auf globale Gerechtigkeit im Rahmen dieser schnellen Verbreitung schnell wieder aus dem Blick. Dies ist insbesondere interessant als der Brundtland-Bericht nach wie vor einer der wichtigsten Referenzpunkte für politische Dokumente mit Nachhaltigkeitsbezug ist, die meist dessen Definition allgemein zitieren, sich aber nicht mit der eigentlichen Problemanalyse und Schwerpunktsetzung des Berichts auseinandersetzen.

3. Fragmentierung und Gleichzeitigkeit in der Gegenwart: Die Übergänge zwischen den Phasen sind fließend und es kommt zu vielen Überlappungen. Trotzdem lässt sich um den Beginn der Finanzkrise der Beginn einer dritten, bis heute dauernden Phase festmachen. Diese zeichnet 
sich weniger durch das Vorherrschen einer dominanten Begrifflichkeit als vielmehr durch die Gleichzeitigkeit unterschiedlicher Stränge und Bedeutungsrahmen aus. Drei davon sind besonders signifikant. a) Der Klimawandel wird zum dominierenden Nachhaltigkeitsproblem. Insbesondere mit dem International Panel on Climate Change (IPCC) entsteht eine starke globale Institution, die den Klimawandel fest oben auf der Agenda und fest in einem Expert*innendiskurs, der v.a. in den sog. Erdsystemforschung geführt wird, etablieren kann. b) Die sogenannte Green Economy. Der Stern-Report für das britische Finanzministerium (Stern 2007) argumentierte, dass die Eindämmung des Klimawandels nicht nur ökologisch, sondern auch ökonomisch sinnvoll sei. Im Zuge der Finanzkrise wurde dieses ökonomische Argument ausgeweitet und z.B. in wesentlich umfassendere Konzeptionen eines „Green New Deal“ (UNEP 2009) überführt. Allgemein wird die Rolle der Ökonomie grundlegend neu interpretiert: Mit der Bioökonomie werden grüne Technologien und Geschäftsmodelle nicht nur umweltfreundlich, sondern auch zum neuen Wachstums- und Wohlstandsmotor. c) Neben diesen beiden dominanten Strömungen finden sich auch Nischen, in denen der politische, an globaler Gerechtigkeit orientierte Nachhaltigkeitsbegriff des Brundtland-Berichts überlebt und weiterentwickelt wird. Diese „Nischen“ finden sich bei den großen UN-Nachhaltigkeitskonferenzen in Johannesburg 2002 und Rio de Janeiro 2012, der Verabschiedung der Millennium Development Goals (2000) und der Sustainable Development Goals (2015). Soziale Bewegungen im globalen Norden und Süden sind weitere wichtige Träger dieses Nachhaltigkeitsbegriffs (z.B. Degrowth-Bewegung, Food Sovereignity Bewegung).

Angesichts dieser knappen Skizze stellt sich die Frage, wie es in einem so hybriden und umstrittenen Feld wie der Nachhaltigkeit überhaupt zu so umfassenden Phasen der Stabilität bzw. zu deren Auf- und Ablösung durch neue dominante Kon- zeptionen kommt. Ist es möglich, Dynamiken und Prozesse zu identifizieren, die ein umfassenderes Verständnis dieser Entwicklungen erlauben?

\section{Stabilisierung und Destabilisierung durch epistemische Politik}

Grundlegend wird davon ausgegangen, dass gegenwärtig keine Gesellschaft unter keinem der aufgezeigten Gesichtspunkte als nachhaltig charakterisiert werden kann und dass sämtliche Praktiken, die sich auf das Konzept der nachhaltigen Entwicklung beziehen, als Praktiken des Suchens und Versuchens zu verstehen sind. Dieses Suchen und Versuchen schließt die oftmals kontrovers umkämpfte - Definition und Stabilisierung der intellektuellen und konzeptionellen Rahmenbedingungen ein. Gleichzeitig beruhen aufgrund der großen Bedeutung geologischer, chemischer und biologischer Prozesse sowie komplexer globaler, sozialer, politischer und ökonomischer Zusammenhänge in diesem Kontext viele dieser Praktiken auf (sehr diversen) hochspezialisierten Wissensbeständen, Expert*innenkulturen und Technologien, wie etwa Computersimulationen, Messsystemen (z.B. von $\mathrm{CO} 2$-Ausstoß oder Energieeinsparungen) in Kombination mit umfassenden Bilanzierungstechnologien, um Nachhaltigkeitsprobleme sichtbar und regelbar sowie mögliche Fortschritte analysierbar zu machen.

In der Folge findet die Auseinandersetzung darüber, welche Herausforderungen eine nachhaltige Gesellschaft zu lösen hätte, wie diese aussehen könnte und welche Wege dorthin führen könnten meist auf einer abstrakt-konzeptionellen Ebene statt. Argumente bedürfen meist einer umfangreichen Datengrundlage sowie der Vermittlung von Expert*innen, die z.B. unterschiedliche Klimadaten in Szenarien anthropogenen Klimawandels übersetzen. Welche Bedeutungen, Handlungen, Strategien und Ordnungselemente als nachhaltig bzw. nachhaltigkeitsförderlich angesehen werden, 
ist somit Gegenstand ,epistemischer Politik' (bzw. Governance). Der politische Charakter dieser Auseinandersetzungen ist darin begründet, dass epistemische Interventionen in der Nachhaltigkeitsdebatte - wie eingangs schon erwähnt - ja selten auf die Schaffung scheinbar neutralen naturwissenschaftlichen Wissens, sondern immer die Rahmenbedingungen für normative Bewertungen und Handlungsmuster beeinflussen wollen. Insofern haben solche Interaktionen in der Regel Konsequenzen, treffen auf Widerstand oder regen die Entstehung alternativer Konzepte und Praktiken an. So verlangte der Übergang von der skizzierten Frühphase zur Entstehung des Konzepts der nachhaltigen Entwicklung vor allem die konzeptionelle Neuverbindung von Ökologie und Entwicklung, um den Widerstand des globalen Südens zu überwinden. Dies gelang zum einen durch die Fokussierung auf globale Gerechtigkeit, die auch einen Ausblick auf eine transformierte Ökonomie ermöglichte. Vor allem verlangte dieser Schritt aber nach Arenenwechsel. Die konzeptionelle Ausrichtung des Brundtland-Berichts auf Gerechtigkeit wäre im wissenschaftlich-systemischen Rahmen der vorhergehenden Phase nicht möglich gewesen, sondern verlangte nach einer Verlagerung der Aktivitäten in die politische Sphäre.

\section{Gesellschaftstheoretische Reflexion der Nachhaltigkeit ${ }^{13}$}

In den Debatten um Nachhaltigkeit wird die Gestaltung einer Gesellschaft thematisiert, in der menschliche Existenz dauerhaft gesichert ist. Obwohl damit Konzepte von Gesellschaft aufgerufen werden, haben sich soziologische Auseinandersetzungen bislang vor allem Teilphänomenen oder einzelne Problem- und Fragestellungen zugewandt. Gewöhnlich werden

13 Nikolai Drews und Nico Lüdtke dabei bestimmte Verständnisse von Nachhaltigkeit vorausgesetzt oder postuliert, ohne dass die gesellschaftstheoretische Bedeutung solcher Annahmen reflektiert würde. Vorgeschlagen wird deshalb, gesellschaftliche Entwicklungen, die im Zusammenhang mit Nachhaltigkeit stehen, aus einer explizit gesellschaftstheoretischen Perspektive zu beleuchten. Dabei gehen wir von den Annahmen der Theorie funktionaler Differenzierung (TfD) aus (Luhmann 1981; Luhmann 1992; Luhmann 1999; Nassehi 2011). In einem ersten Teil wird beleuchtet, wie Nachhaltigkeit aus Sicht der TfD als ein Phänomen beschrieben werden kann, dass eine stabilisierende Funktion für gesellschaftliche Ausdifferenzierung leisten kann. Der zweite Teil widmet sich, im Horizont kritischer Stimmen zur TfD, der Frage nach möglichen Entdifferenzierungserscheinungen.

Nachhaltigkeit, so lässt es sich beobachten, hat über die gesamte Gesellschaft hinweg fast überall Eingang in die Kommunikation gefunden. Die Nachhaltigkeitssemantik tritt oftmals dann in Erscheinung, wenn bestimmte Handlungsweisen oder Einrichtungen als nicht-nachhaltig thematisiert und kritisiert werden. So etwa, wenn die anscheinend rücksichtslose Ausbeutung einer Ressource angeprangert wird oder der dauerhafte Erhalt bestimmter materieller Sachverhalte - wie z.B. Ökosysteme - gefordert wird. Zudem scheint letztlich der Erhalt der menschlichen Existenz Kern der Debatte zu sein, auch wenn dieser nicht immer expliziert wird. Vor diesem Hintergrund lässt sich die These formulieren, dass Nachhaltigkeit als eine Institution der funktional differenzierten Gesellschaft beschreibbar ist, welche die Gesellschaftsstruktur hinsichtlich des Erhalts materieller Umwelten stabilisiert.

In einem frühen Werk (Luhmann 1965) beschreibt Luhmann einen ähnlichen Mechanismus, wenn er die Funktion der Grundrechte für die moderne Gesellschaft rekonstruiert. Dabei erweitert er das rechtswissenschaftliche, grundrechtsdog- 
matische Verständnis von Grundrechten in der Hinsicht, dass er aus soziologisch informierter Perspektive aufzeigt, wie die Grundrechte nicht allein das Verhältnis von Bürger*in und Staat konstituieren, sondern vielmehr die gesamte Sozialordnung der funktionalen Differenzierung in ihrem Verhältnis zum modernen Individuum institutionalisieren. Dabei handelt es sich um eine zweiseitige Problematik: Einerseits wird über normierte Freiheitsrechte gewährleistet, dass jeder einzelne in den verschiedenen, differenzierten Funktionssystemen teilhaben kann, indem die Möglichkeit des Zugangs zu diesen Kommunikationszusammenhängen freigehalten wird. So garantiert beispielsweise der Eigentumsschutz grundsätzlich die Teilnahme an ökonomischen Kommunikationszusammenhängen - ohne damit etwa eine konkrete Ausstattung mit Gütern zu gewähren. Andererseits wird über den Begriff und institutionalisierten Wert der Würde das Individuum vor einer Vereinnahmung seiner Person durch einen einzelnen Funktionszusammenhang geschützt. Das Individuum bleibt damit offen und kommunikationsfähig für verschiedene funktionale Zusammenhänge der Gesellschaft.

In Analogie dazu ist mit Blick auf Nachhaltigkeit die Bedeutung materieller Sachverhalte zu betonen, auf die in der Debatte stark fokussiert wird. Die Luhmannsche Konstruktion des Verhältnisses von Individuum und funktionaler Differenzierung im Rahmen der Grundrechte bezieht sich vor allem auf den Erhalt von Kommunikationschancen. Demgegenüber ist die These, dass die sich der Gesellschaft zunehmend aufdrängende Relevanz ökologischer Probleme und die Thematisierung von Nachhaltigkeit darauf hinweisen, dass eine vergleichbare Institution hervorgebracht wird, wie bei den Grundrechten. Das Spezifische dabei stellt der schwerpunkthafte Bezug zum Materiellen dar. Innerhalb der modernen Gesellschaft gelten üblicherweise Menschen als legitime Mitglieder dieser Gesellschaft (Lindemann 2014, mit Bezug auf Systemtheorie
Lindemann 2009; Henkel 2016b). Allerdings ist der Mensch mehr als nur ein Bewusstseinssystem, das eine relevante Umwelt des Kommunikationssystems Gesellschaft darstellt. Der Mensch verfügt gleichsam über einen Körper, mit dem er Teil der materiellen Welt ist. Solch ein verkörperter Mensch ist angewiesen auf materielle Umwelten, er kann überhaupt nur in einem Verweisungszusammenhang zu einer entsprechenden Umwelt auftreten. Die Zweiseitigkeit der Problematik, auf welche die angenommene Institution der Nachhaltigkeit reagiert, lässt sich nun wie folgt darstellen: Einerseits dürfen für das Aufrechterhalten der gesellschaftlichen Differenzierung materielle Umwelten des Menschen nicht von einzelnen Kommunikationszusammenhängen der Gesellschaft für sich vereinnahmt werden, da sie ansonsten für Bezüge anderer Zusammenhänge nicht hinreichend frei sind. Andererseits darf auch der Mensch als verkörpertes Wesen in seinem Zugang zu materiellen Umwelten nicht zu sehr beschränkt werden, da ihm sonst die Teilnahme an den differenzierten Kommunikationszusammenhängen nicht gelingt. Sorgt die funktional differenzierte Gesellschaft nicht für einen freien Zugriff auf relevante materielle Umwelten und lässt sie etwa einer einseitigen Vereinnahmung materieller Umwelten - beispielsweise als restlos auszubeutende Ressourcenreservoirs - freien Lauf, so gefährdet sie ihre weitere Differenzierung.

In dieser Hinsicht lässt sich eine integrative Funktion von Nachhaltigkeit im Rahmen der differenzierten Gesellschaft darin sehen, dass über die Betonung eines gemeinsamen Bezugspunkts - verkörperter Mensch - ein über die verschiedenen Funktionssysteme hinweg geteilten Maßstab aufgestellt wird, an dem sich Zugriffe auf Umwelten messen lassen müssen. Damit sind bestimmte Grenzen impliziert, die nicht überschritten werden dürfen, wenn es um den Erhalt der modernen Gesellschaft und damit auch der Menschheit geht. Einseitige Vereinnahmungen materieller Umwelten stellen in diesem 
Sinne eine gesamtgesellschaftliche Gefährdung dar, die als reflexives Moment - was unter der Bezeichnung ,Nachhaltigkeit' auftritt - Eingang in gesellschaftliche Strukturen findet.

In Verbindung mit dem allgemeinen gesellschaftlichen Bedeutungsgewinn von Nachhaltigkeit steht im Besonderen auch die Ausweitung einer Nachhaltigkeitsorientierung im Bereich der Wissenschaft (vgl. Abschnitt 3). Aus der Perspektive der TfD lässt sich danach fragen, ob sich dieser Prozess als Hinweis auf Veränderungen in den Relationen von Wissenschaft, Politik, Wirtschaft und Zivilgesellschaft deuten lässt. Die Verbreitung nachhaltigkeitsorientierter transdisziplinärer Projekte wirft in dieser Perspektive die Frage der trennscharfen Unterscheidung von Funktionssystemen auf. Vor dem Hintergrund der Annahme, dass die ausdifferenzierten gesellschaftlichen Subsysteme getrennt voneinander operieren, stellt sich das Problem, inwieweit sich die Veränderungen als Entdifferenzierungserscheinungen begreifen lassen.

Den Ausgangspunkt bildet zum einen die Beobachtung zunehmender Initiativen einer wissenschaftspolitischen Umgestaltung des Wissenschaftssystems. Im Kern geht es dabei um eine Übersetzung der internationalen Diskussion um Nachhaltigkeit in nationale Wissenschaftspolitiken (Kates et al. 2001). Diese Ansätze zielen darauf, dass die Wissenschaft nicht nur zunehmend gefordert sei, gesellschaftlich relevante Probleme innerhalb der Forschung zu adressieren. Gefordert wird ebenfalls, gesellschaftlichen Wandel aktiv mitzugestalten. Forschende sollen als verantwortliche Akteure zur Lösung großer gesellschaftlicher Herausforderungen beitragen (WGBU 2011; Wissenschaftsrat 2015). Die förderpolitischen Umsetzungen solcher Forderungen (wie etwa das BMBF-Rahmenprogramm „Forschung für Nachhaltige Entwicklungen“ (FONA) haben zu einer beachtlichen Veränderung der Forschungslandschaft geführt.
Ferner ist $\mathrm{zu}$ beobachten, dass sich innerhalb der Forschung selbst Veränderungen ergeben haben - und zwar zeitlich früher als im wissenschaftspolitischen Diskurs. Im Bereich der außeruniversitären Forschung hat sich ein neuer Forschungsmodus herausgebildet, der mittlerweile zunehmend und auch über diesen Bereich hinaus Anwendung findet. Kennzeichen dieses neuen Typs der Forschung ist einerseits ein spezifischer Zuschnitt auf der Sachebene sowie andererseits eine bestimmte Form, wie Forschungsprozesse innerhalb von Projekten sozial organisiert werden. Die Verbindung aus Problemorientierung und partizipativer Form der Organisation der Wissenserzeugung ergeben das Spezifikum dieses neuen Typs. „Transdisziplinarität“ hat sich hierbei als Terminus etabliert, sowohl für eine inhaltliche Ausrichtung auf komplexe gesellschaftliche Problemstellungen als auch hinsichtlich der Umstellung auf eine kooperative Form der Wissenserzeugung, die außerwissenschaftliche Akteure, Wissensformen, Werthaltungen, Interessen und Ansprüche in den Forschungsprozess mit einbindet (Hadorn Hirsch et al. 2008, Jahn et al. 2012). Der Begriff wird maßgeblich - wenn auch nicht ausschließlich - im Zusammenhang mit „Nachhaltigkeit“ verwendet.

Zur gesellschaftstheoretischen Interpretation lässt sich an die Diskussion zur TfD anschließen, die im Gespräch zwischen der Systemtheorie und der empirischen Wissenschaftsforschung entstanden ist. Entsprechend der Fokussierung auf den operativen und ereignishaften Charakter der Systembildung durch das Theorem der symbolisch generalisierten Kommunikationsmedien steht hierbei die Frage im Mittelpunkt, auf welche Weise Operationen unterschiedliche Anschlusszusammenhänge herstellen und damit eine ausdifferenzierte Ordnung hervorbringen. Die These von Knorr-Cetina (1992) ist, dass Kommunikationen im Feld der Wissenschaft nicht ausschließlich oder vorwiegend eine wahr-falsch Codierung aufweisen; stattdessen lasse sich em- 
pirisch beobachten, dass eine Reihe von Kriterien relevant ist, die nicht-wissenschaftlich sind. AuBerdem sei die Annahme infrage zu stellen, dass es nur die Entwicklungstendenz steigender Spezialisierung und Differenzierung der Teilsysteme gäbe. Aus Sicht der Systemtheorie hält Nassehi die These entgegen, dass nicht die empirisch vorfindlichen Kommunikationen im „Labor“ für die Frage maßgeblich sind, wie in wissenschaftlicher Kommunikation Anschlussfähigkeit hergestellt wird: „Nicht die Routinen der Forschung und ihre pralle Praxis machen den Anschlusszusammenhang des Wissenschaftssystems aus, sondern eine bestimmte Form der Präsentation von Forschung in Publikationen“ (Nassehi 2004: 107).

Vor dem Hintergrund dieser Diskussion lassen sich die Veränderungen im Wissenschaftssystem als ein neuer Phänomenkomplex deuten, der einer gesellschaftstheoretischen Interpretation bedarf. Zugleich ergeben sich empirische Irritationen für den bestehenden Theorierahmen der TfD. Die beschriebenen Entwicklungen lassen sich als Hinweise auf Prozesse der Entdifferenzierung und Entspezialisierung deuten. Im Bereich transdisziplinärer Forschung, die gleichzeitig problem- bzw. anwendungsorientiert ist und partizipativ realisiert wird, scheinen sich Veränderungen in der Selbstbeschreibung der Forschung zu ergeben, insofern nicht allein das symbolisch generalisierte Kommunikationsmedium wissenschaftlicher Wahrheit in Anspruch genommen wird. Diese Tendenzen zeichnen sich sogar stärker ab, als dies bei den Laborstudien im Feld der „klassischen“ disziplinär organisierten und dekontextualisierenden Forschung der Fall war.

Insgesamt zeigt sich, dass mittels der Explikation gesellschaftstheoretischer Annahmen ein Rahmen eröffnet wird, um die gegenwärtigen Phänomene, die in Verbindung mit der Debatte um Nachhaltigkeit stehen, in ihrer geschichtlichen und gesellschaftlichen Bedeutung einzuordnen und $\mathrm{zu}$ verstehen. Wir haben Wege aufgezeigt, wie die TfD in dieser Hinsicht fruchtbar gemacht werden kann. Dabei zeigt sich, dass auch die Theorie selbst auf Herausforderungen stößt. Das Potenzial zur gesellschaftstheoretischen Reflexion dieser Theorieoption lässt sich dann ausschöpfen, wenn die empirischen Irritationen im Zuge des Bedeutungsgewinns von „Nachhaltigkeit“ produktiv aufgenommen werden können und Anstoß zur Weiterentwicklung geben.

\section{Ausblick: Reflektierte Norma- tivität, Gesellschaft und die Stärke der Pluralität ${ }^{14}$}

Die Soziologie befasst sich mit zentralen Kernproblemen der Nachhaltigkeitsproblematik und entwickelt seit kurzem Ansätze, soziologische Theorie für eine Reflektion auf Nachhaltigkeit und die Entwicklung von Nachhaltigkeit als dezidiert soziologischen Gegenstand anzuwenden.

Jedoch werden im gesellschaftlichen Diskussionsprozess $\mathrm{zu}$ Nachhaltigkeit soziologische Sichtweisen zu diesem Themenkomplex bislang kaum wahrgenommen - es besteht neben dem Synthese- auch ein Sichtbarkeitsproblem. Trotz einzelner Vorreiter bleibt so die Beobachtung von Brand aus den 1990er Jahren gültig, dass sich die Soziologie gegenüber der Nachhaltigkeitsdebatte dezidiert abgrenzt (Brand 1997). Natur, Technosphäre, sozialer Eigensinn, Wandel, normative Kritik, Wissen, Risiko, Governance und Entdifferenzierung - all diese in der Nachhaltigkeitsdebatte relevanten Aspekte kommen in der Soziologie vor, doch werden sie kaum für diese Debatte fruchtbar gemacht.

Die „Soziologie der Nachhaltigkeit“ kann entsprechende Perspektiven verbinden und die kritische Reflektion auf implizite Prämissen

14 Anna Henkel 
der Nachhaltigkeitsdebatte gerade mit Blick auf eine „nachhaltige“ Handlungsorientierung reflektieren. Eine Einbeziehung soziologischer Perspektiven auf Nachhaltigkeit über verschiedene Diskussionsstränge hinweg kann dazu beitragen, eine dringend erforderliche Reflexionsdimension in die Debatte um Nachhaltigkeit einzuführen. Ausgehend von diesem Befund wurde als spezifische Herausforderung der Soziologie festgestellt, sich zu der Problemorientierung sowie dem normativen Charakter der Nachhaltigkeit zu positionieren. Als Beiträge zur Nachhaltigkeitsdebatte können solche Ansätze gesehen, zueinander verortet und miteinander ins Gespräch gebracht werden, die sich zur Gleichzeitigkeit von Beobachtungs- und Transformationsorientierung verhalten und - ob mit bereichsspezifischem oder sozialtheoretischem Fokus - beide Orientierungen integrieren.

Die Stärke der Soziologie liegt - wie im Kontext einer Diskussion um eine Soziologie der Nachhaltigkeit näher auszuarbeiten - vermutlich gerade in der Vielfältigkeit. Im Sinne eines Ausblicks lassen sich vor allem drei Aspekte für die weitere Diskussion um eine Soziologie der Nachhaltigkeit ausmachen: Erstens gilt es, implizite Prämissen der Nachhaltigkeitsdebatte zu explizieren, um durch Reflektion auf den normativen Charakter der Nachhaltigkeitsdebatte sowohl der Soziologie die Möglichkeit zu geben, sich unbefangen diesem Themenfeld zu widmen; sowie zugleich die Nachhaltigkeitsdebatte selbst gesellschaftlich zu verorten. Zweitens gilt es, soziologische Zugänge und Konzepte auf ihren konkreten, potentiellen Beitrag zur Nachhaltigkeitsdebatte hin zu überprüfen. Drittens schließlich können durch eine vergleichende Diskussion unterschiedlicher soziologischer Konzepte die Konturen eines soziologischen Nachhaltigkeitskonzepts entwickelt werden. Die Identifizierung der Herausforderung, Beobachtungs- und Transformationsorientierung mit unterschiedlichem Grad reflexiver Distanz $\mathrm{zu}$ integrieren ist ein Vorschlag zur Spezifikation der „Landschaft“ einer Soziologie der Nachhaltigkeit, in der sich Ansätze mit unterschiedlicher bereichs- oder gesellschaftstheoretischer Fokussierung kartieren lassen.

\section{Literatur}

Agrawal, A./Lemos, M./Orlove, B./Ribot, J. (2012): Cool heads for a hot world - Social sciences under a changing sky. In: Global Environmental Change, 22. Jg., Heft. 2, S. 329-331.

Baranek, E./Günther, B. (2005): Erfolgsfaktoren von Partizipation in Naturschutzgroßprojekten-Das Beispiel: Moderationsverfahren im Gewässerrandstreifenprojekt Spreewald. In: Feindt, P./Newig, J. [Hrsg.]: Partizipation, Öffentlichkeitsbeteiligung, Nachhaltigkeit. Perspektiven der Politischen Ökonomie. Metropolis: Marburg.

Barry, J. (2012): The Politics of Actually Existing Unsustainability: Human Flourishing in a Climate-Chaned, Carbon Constrained World. Oxford Scholarship: Oxford.

Beck, U. (1986): Risikogesellschaft. Auf dem Weg in eine andere Moderne. Suhrkamp: Frankfurt.

Beck, U. (2014): Sinn und Wahnsinn der Moderne. Laudatio auf Zygmunt Bauman. Soziologiekongress. Trier.

Berger, P./Luckmann, T. (1980): Die gesellschaftliche Konstruktion der Wirklichkeit. Eine Theorie der Wissenssoziologie. Fischer: Frankfurt

Bingham, G, (1986): Resolving Environmental Disputes. A Decade of Experience. Conservation Foundation: Washington.

Bora, A./Henkel, A./Reinhardt, C. [Hrsg.] (2014): Wissensregulierung und Regulierungswissen. Velbrück: Weilerswist.

Böschen, S. (2016): Hybride Wissensegime. Skizze einer soziologischen Feldtheorie. Nomos: Baden-Baden. 
Bosso, C. (1987): Pesticides and Politics. The Life Cycle of a Public Issue. Pittsburgh University Press: Pittsburgh, PA.

Bourdieu, P. (1998): Vom Gbrauch der Wissenschaft. Für eine klinische Soziologie des wissenschaftlichen Feldes. UVK: Konstanz.

Brand, K.-W. [Hrsg.] (1997): Nachhaltige Entwicklung. Eine Herausforderung an die Soziologie. Leske+Budrich: Opladen.

Brand, K.-W. (2002): Politik der Nachhaltigkeit. Nachhaltigkeitsforschung in der Helmhotz-Gemeinschaft. Edition Sigma: Berlin.

Brand, K.-W. (2014): Umweltsoziologie: Entwicklungslinien, Basiskonzepte und Erklärungsmodelle. Beeltz Juventa: Weinheim/München.

Brinkmann, C./Bergmann, M./Rödder, S./ Schuck-Zöller, S. (2015): Zur Integration von Wissenschaft und Praxis als Forschungsmodus - ein Literaturüberblick. In: Climate Service Center 2.o., Bericht 23: Hamburg.

Bronfenbrenner, U. (1981): Die Ökologie der menschlichen Entwicklung. Natürliche und geplante Experimente. Klett-Cotta: Stuttgart.

Büscher, C./Sumpf, P. (2015): "Trust" and "confidence" as socio-technical problems in the transformation of energy systems. In: Energy, Sustainability and Society, 5. Jg., Heft 34. Online: DOI 10.1186/s1370513015-10063-13707.

Buttel, F. (1976): Social Science and the Environment: Competing Theories. In: Social Science Quarterly, Heft 57, S. 307-323.

Catton, W./Dunlap, R. (1978): Environmental Sociology: A new Paradigm. In: The American Sociologist, Heft 13, S. 41-49.

Coglianese, C. (1997): Assessing Consensus: The Promise And Performance Of Negotioated Rule-making. In: Duke Law Journal, Heft 46, S. $1255^{-1346 . ~}$
Conrad, J. (2005): Grüne Gentechnik - Gestaltungschance und Entwicklungsrisiko: Perspektiven eines regionalen Innovationsnetzwerks. Deutscher Universitätsverlag: Wiesbaden.

Descola, P. (2011): Jenseits von Natur und Kultur. Suhrkamp: Frankfurt am Main.

DFG (2013): Exzellenzinitiative auf einen Blick. Der Wettbewerb des Bundes und der Länder zur Stärkung der universitären Spitzenforschung. Berlin: BMBF.

Diekmann, A./Jaeger, C. [Hrsg.] (1996): Umweltsoziologie. In: Sonderheft 36 der Kölner Zeitschrift für Soziologie und Sozialpsychologie. Westdeutscher Verlag: Opladen.

Edwards, P. (2004): Infrastructure and modernity: Force, time and social organization in the history of sociotechnical systems. In: Misa , T./Brey, P./Feenberg, A. [Hrsg.], Modernity and Technology. MIT-press: Cambridge, MA, S. 185-225.

Etzioni, A. (1996): Die faire Gesellschaft. Jenseits von Sozialismus und Kapitalismus. Suhrkamp: Frankfurt am Main.

Etzioni, A. (1997): Die Verantwortungsgesellschaft. Individualismus und Moral in der heutigen Demokratie. Campus Verlag: Frankfurt am Main, New York.

Feindt, P./Newig, J. (2005): Partizipation, Öffentlichkeitsbeteiligung, Nachhaltigkeit. Perspektiven der politischen Ökonomie. Metropolis: Marburg.

Firnenburg, L./Görgen, B./Grundmann, M./ Wendt, B. (2017): Doing Sustainability Die soziale Konstruktion der Nachhaltigkeit zwischen mikro- und makrostruktureller Praxis. In: Lessenich, S. [Hrsg.] (2017): Geschlossene Gesellschaften. Verhandlungen des 38. Kongresses der Deutschen Gesellschaft für Soziologie in Bamberg 2016 (im Erscheinen)

Fligstein, N./McAdam, D. (2012): A theory of fields. Oxford University Press: New York. 
Geis, A. (2005): Regieren mit Mediation: Das Beteiligungsverfahren zur zukünftigen Entwicklung des Frankfurter Flughafens. In: Studien zur politischen Gesellschaft, Band 6. Verlag für Sozialwissenschaften: Wiesbaden.

Gibbons, M./Limoges, C./Nowotny, H./ Schartzman, S./Scott, P./Trow, M. (1994): The New Production of Knowledge. The Dynamics of Science and Research in Contemporary Societies. Sage: London.

Görgen, B./Wendt, B. (2015): Nachhaltigkeit als Fortschritt denken. Grundrisse einer soziologisch fundierten Nachhaltigkeitsforschung. In: Soziologie und Nachhaltigkeit - Beiträge zur sozialökologischen Transformationsforschung, 1. Jg., Heft 1. Online: https://wwu.de/Ejournals/index.php/sun/ article/view/1443 [Zugriff: 14.12.2016].

Groß, M. (2001): Die Natur der Gesellschaft. Eine Geschichte der Umweltsoziologie. Juventa: Weinheim.

Groß, M. [Hrsg.] (2011): Handbuch Umweltsoziologie. VS Verlag: Wiesbaden.

Grundmann, M. (1999): Konstruktivistische Sozialisationsforschung. Suhrkamp: Frankfurt am Main.

Grundmann, M. (2013): Humanökologie, Sozialstruktur und Sozialisation. In: Hurrelmann, K./Grundmann, M./Walper, S. [Hrsg.]: Handbuch Sozialisationsforschung. Beltz: Weinheim.

Grundmann, M. (2016): Gemeinsam - nachhaltig. Argumente für eine sozialisationstheoretische Bestimmung sozialer Nachhaltigkeit. Soziologie und Nachhaltigkeit - Beiträge zur sozialökologischen Transformationsforschung, 2. Jg., Heft 5. Online: https:// www.uni-muenster.de/Ejournals/index. $\mathrm{php} / \mathrm{sun} /$ article/view/1756 [Zugriff: 06.02.2017].

Grundmann, M./Kunze, I. (2008): Systematische Sozialraumforschung. Urie
Bronfenbrenners Ökologie der menschlichen Entwicklung und die Modellierung mikrosozialer Raumgestaltung. In: Kessel, F./Reutlinger, C. [Hrsg.]: Schlüsselwerke der Sozialraumforschung. Traditionslinien in Text und Kontexten. Springer VS: Wiesbaden, S. 172-188.

Grundmann, R. (2016): Climate change as a wicked social problem. In: Nature Geoscience, 9. Jg., Heft 8, S. 562-563.

Grunwald, A. (2016): Nachhaltigkeit verstehen. Arbeiten an der Bedeutung nachhaltiger Entwicklung. Oecom: München.

Häberli， R./Grossenbacher-Mansuy， W./Klein, J. (2001): Summary. In: Klein, J./Grossenbacher-Mansuy, W./Häberli, R./Bill, A./Scholz, R./Welti, M. [Hrsg.]: Transdisciplinarity. Joint problem solving among science, technology, and society: an effective way for managing complexity. Boston: Basel, S. 3-5.

Hirsch Hadorn, G./Biber-Klemm, S./Grossenbacher-Mansuy, W./Hoffmann-Riem, H./ Joye, D./Pohl, C./Wiesmann, U./Zemp, E. (2008): Emergence of Transdisciplinarity as a Form of Research. In: Hadorn Hirsch, G./Hoffmann-Riem, H./Biber-Klemm, S./Grossenbacher-Mansuy, W./Joye， D./ Pohl, C./Wiesmann, U./Zemp, E. [Hrsg.]: Handbook of Transdisciplinary Research. Springer: Wiesbaden, S. 19-39.

Hampel, J. (1999): Gentechnik in der Öffentlichkeit: Wahrnehmung und Bewertung einer umstrittenen Technologie. Campus-Verlag: Frankfurt.

Henkel, A. (2013/2014): Gesellschaftstheorie der Verantwortung. Funktion und Folgen eines Mechanismus der Reduktion sozialer Komplexität. In: Soziale Systeme, Sonderhaft „Precarious Responsibility“, 19. Jg., Heft 2, S. 471-501.

Henkel, A. (2016a): Natur, Wandel, Wissen. Beiträge der Soziologie zur Debatte um 
nachhaltige Entwicklung. In: Soziologie und Nachhaltigkeit - Beiträge zur sozialökologischen Transformationsforschung, 2. Jg., Heft 1. Online: https://www.uni-muenster. de/Ejournals/index.php/sun/article/ view/1675 [Zugriff: 06.02.2017].

Henkel, A. (2016b): Posthumanism, the Social and the Dynamics of Material Systems. In: Theory, Culture \& Society, DOI: 10.1177/0263276415625334.

Henkel, A./Åkerstrøm-Andersen, N. (2013/2014): Precarious Responsibility. In: Soziale Systeme, Sonderheft.

Honneth, A. (1995): Einleitung. In: Honneth, Axel [Hrsg.]: Kommunitarismus. Eine Debatte über die moralischen Grundlagen moderner Gesellschaften. Campus: Frankfurt am Main.

Jahn, T./Bergmann, M./Keil, F. (2012): Transdisciplinarity: Between mainstreaming and marginalization. Ecological Economics 79, 1-10

Kaldewey, D. (2013): Tackling the Grand Challenges. Reflections on the Responsive Structure of Science. In: EU-SPRI Conference: Science dynamics and research systems. The role of research in meeting societal challenges, Madrid.

Kaldewey, D. (2017): Von Problemen zu Herausforderungen: Ein neuer Modus der Konstruktion von Objektivität zwischen Wissenschaft und Politik. In: Stephan Lessenich (Hg.) Geschlossene Gesellschaften. Verhandlungen des 38. Kongresses der Deutschen Gesellschaft für Soziologie in Bamberg 2016. Online: http://publikationen.soziologie.de [Zugriff: 13.03.2017].

Kates, R./Clark, W./ Hall, M./ Jaeger, C./Lowe, I./McCarthy, J./ Schnellnhuber, H./Bolin, B./Dickson, N./Faucheux, S./Gallopin, G./ Grübler, A./Huntley, B./Jäger, J./Jodha, N./Kasperson, R./Mabogunje, A./ Matson, P./Mooney, H./Moore, B./O’Riordan, T./
Svedin, U. (2001): Sustainable Science. In: Science, 292. Jg., Heft 5517, S. 641-642.

Knorr Cetina, K. (1981): The Manufacture of Knowledge. An Essay on the Constructivist and Contextual Nature of Science. Pergamon Press: Oxford.

Knorr Cetina, K. (1992): Zur Unterkomplexität der Differenzierungstheorie. Empirische Anfragen an die Systemtheorie. In: Zeitschrift für Soziologie, 21. Jg., Heft 6, S. 406-419.

Kowol, U. (1998): Innovationsnetzwerke: Ein Modell der Technikgenese. Deutscher Universitätsverlag: Wiesbaden.

Latour, B./Woolgar, S. (1986): Laboratory Life. The Construction of Scientific Facts. Princeton University Press: Princeton, NJ.

Lewin, K. (1963): Feldtheorie in den Sozialwissenschaften. Verlag Hans Huber: Stuttgart.

Lindemann, G. (2009): Das Soziale von seinen Grenzen her denken. Velbrück Wissenschaft: Weilerswist.

Lindemann, G. (2014): Weltzugänge. Die mehrdimensionale Ordnung des Sozialen. Velbrück: Weilerswist.

Lockie, S./Sonnenfeld, D./Fisher, D. [Hrsg.] (2014): Routledge International Handbook of Social and Environmental Change. Routledge.

Lucke, D. (1997): Akzeptanz. In: Gottwald, W. [Hrsg.]: Außergerichtliche Konfliktregelung. AKR-Handbuch für Rechtsanwälte und Notare. Luchterhand: Neuwied, S. 1-10.

Lüdtke, N. (2015): Das Soziale ohne Grenzen? Eine historisch-wissenssoziologische Analyse zu den Grenzen der Sozialwelt in der Frühen Neuzeit. Velbrück Wissenschaft: Weilerswist.

Luhmann, N. (1965): Grundrechte als Institution. Duncker \& Humblot: Berlin.

Luhmann, N. (1981): Gesellschaftsstruktur und Semantik. Studien zur Wissenssoziologie der modernen Gesellschaft. Suhrkamp: Frankfurt am Main. 
Luhmann, N. (1986): Ökologische Kommunikation. Kann die moderne Gesellschaft sich auf ökologische Gefährdungen einstellen? Westdeutscher Verlag: Opladen.

Luhmann, N. (1992): Beobachtungen der Moderne. Westdeutscher Verlag: Opladen.

Luhmann, N. (1999): Die Gesellschaft der Gesellschaft. Suhrkamp: Frankfurt am Main.

Maasen, S./Lengwiler, M./Guggenheim, M. (2006): Practices of Transdisciplinary Research: Close(r) Encounters of Science and Society. Introduction to Science \& Public Policy Special Issue on Transdisciplinarity. In: Science \& Public Policy, 33. Jg., Heft 6, S. 394-398.

Meadows, D./Meadows, D./Randers, J./Behrens, W. (1972): The limits to growth. A report for the Club of Rome's project on the predicament of mankind. Universe Books: New York.

Nassehi, A. (2004): Die Theorie funktionaler Differenzierung im Horizont ihrer Kritik. In: Zeitschrift für Soziologie, 33. Jg., Heft 2: S. 98-118.

Nassehi, A. (2011): Gesellschaft der Gegenwarten. Suhrkamp: Berlin.

Neckel, S. (2015): Burnout. Das gesellschaftliche Leid der Erschöpfung. In: Transit. Europäische Revue, 46. Jg. (Winter 2014/15), S. 116-130.

Neckel, S./Wagner, G. [Hrsg.] (2013): Leistung und Erschöpfung. Burnout in der Wettbewerbsgesellschaft. Suhrkamp: Berlin.

Newig, J./Fritsch, O. (2009): Der Beitrag zivilgesellschaftlicher Partizipation zur Effektivitätssteigerung von Governance. Eine Analyse umweltpolitischer Beteiligungsverfahren im transatlantischen Vergleich. In: Bode, I./Evers, A./Klein, A. [Hrsg.]: Bürgergesellschaft als Projekt. Eine Bestandsaufnahme zu Entwicklung und Förderung zivilgesellschaftlicher Potenziale in Deutschland. Verlag für Sozialwissenschaften: Wiesbaden, S. 214-239

Newig, J./Fritsch, O. (2011): Ökologische Nachhaltigkeit durch zivilgesellschaftliche Partizipation? Zum Stand der Debatte in der internationalen Literatur. In: Handler, M./Trattnigg, R. [Hrsg.]: Zukunft der Öffentlichkeitsbeteiligung. Chancen. Grenzen. Herausforderung. Bundesministerium für Land- und Forstwirtschaft, Umwelt und Wasserwirtschaft: Online: www.partizipation.at [Zugriff: 05.01.2017], S. 55-64.

Nowotny, H. (2005): Science and Society: Highand Low-Cost Realities for Science and Society. In: Science, 308. Jg., Heft 5725, S. 1117-1118.

Nowotny, H./Scott, P./Gibbons, M. (2001): Re-Thinking Science. Knowledge and the Public in an Age of Uncertainty. Polity Press: Cambridge.

Pfister, T./Schweighofer, M./Reichel, A. (2016): Sustainability. Routledge: London.

Pieper, R. (1979): Wissensformen und Rechtfertigungsstrategien. Ein Beitrag zum Vermittlungsproblem zwischen Wissenschaft, Technik und Alltag. In: Soziale Welt, 30. Jg., Heft 1, S. 50-69.

Proctor, R./Schiebinger, L. [Hrsg.] (2008): Agnotoloy. The Making und Unmaking of Ignorance. Stanford UP: Stanford.

Rammert, W. (2007): Technik - Handeln - Wissen. $\mathrm{Zu}$ einer pragmatistischen Technik- und Sozialtheorie. VS Verlag für Sozialwissenschaften: Wiesbaden

Rammert, W./Schubert, C. (2006): Technografie. Zur Mikrosoziologie der Technik. Campus: Frankfurt.

Renn, O./Kastenholz, H./Schild, P./Webler, T./Wilhelm, U. (1998): Der kooperative Diskurs in der Praxis: Standortsuche für eine Deponie im Kanton Aargau. In: Renn, O./Kastenholz, H./Schild, P./Webler, T./ Wilhelm, U. [Hrsg.]: Abfallpolitik im ko- 
operativen Diskurs: Bürgerbeteiligung bei der Standortsuche für eine Deponie im Kanton Aargau. Hochschulverlag: Zürich, S. 107-131.

Ribot, J. (2014): Cause and response: vulnerability and climate in the Anthropocene. In: The Journal of Peasant Studies, 41. Jg., Heft 5, S. 667-705.

Schuppert, G./Voßkuhle, A. (2008): Governance von und durch Wissen. Nomos: Baden-Baden.

Schützeichel, R. [Hrsg.] (2007): Handbuch Wissenssoziologie und Wissensforschung. UVK Verlagsgesellschaft: Konstanz.

Stehr, N./von Storch, H. (1995): The social construct of climate and climate change. In: Climate Research 5. Jg., S. 989-105.

Stern, N. (2007): The Economics of Climate Change: The Stern Review. Cambridge University Press: Cambridge.

Striegnitz, M. (1995): Das Münchehagen-Verfahren. In: Hofmann-Dally, A./Weidner, H./Fietkau, H.-J. [Hrsg.]: Mediation als politischer und sozialer Prozess. Evangelische Akademie Loccum: Rehburg-Loccum, S. 9-32.

Tomasello, M. (2016): Eine Naturgeschichte der menschlichen Moral. Suhrkamp: Frankfurt am Main.

Ullrich, C. (2008): Die Akzeptanz des Wohlfahrtsstaates: Präferenzen, Konflikte, Deutungsmuster. Verlag für Sozialwissenschaften: Wiesbaden.

UNEP - United Nations Environment Programme (2009): Global Green New Deal. Policy Brief. Nairobi.

WBGU - Wissenschaftlicher Beirat der Bundesregierung Globale Umweltveränderungen (2011): Welt im Wandel: Gesellschaftsvertrag für eine Große Transformation. Hauptgutachten 2011. WBGU: Berlin.
WCED - World Commission on Envirinment and Development (1987). Our Common Future. New York.

Wehling, P. (2006): Im Schatten des Wissens? Perspektiven der Soziologie des Nichtwissens. UVK: Konstanz.

Wehling, P. (2007): Wissensregime. In: Schützeichel, R. [Hrsg.]: Handbuch der Wissenssoziologie und Wissensforschung. UVK: Konstanz, S. 704-712.

Wendt, B. (2017): Nachhaltigkeit als Utopie. Dissertation. Münster

Wissenschaftsrat (2015): Zum wissenschaftspolitischen Diskurs über große gesellschaftliche Herausforderungen. Positionspapier. Hrsg. v. Wissenschaftsrat. Online: http:// www.wissenschaftsrat.de/download/ archiv/4594-15.pdf [Zugriff: 05.12.2016].

Würtenberger, T.(1996): Die Akzeptanz von Verwaltungsentscheidungen. Verwaltung 2000. Nomos: Baden-Baden. 